University of Wollongong

Research Online

Faculty of Engineering and Information

Faculty of Engineering and Information

Sciences - Papers: Part A

Sciences

$1-1-1998$

Completeness of the propositions-as-types interpretation of intuitionistic logic into illative combinatory logic

\author{
Wil Dekkers \\ Catholic University, Netherlands \\ Martin Bunder \\ University of Wollongong, mbunder@uow.edu.au \\ Henk Barendregt \\ Catholic University, Netherlands
}

Follow this and additional works at: https://ro.uow.edu.au/eispapers

Part of the Engineering Commons, and the Science and Technology Studies Commons

Research Online is the open access institutional repository for the University of Wollongong. For further information contact the UOW Library: research-pubs@uow.edu.au 


\title{
Completeness of the propositions-as-types interpretation of intuitionistic logic into illative combinatory logic
}

\begin{abstract}
Illative combinatory logic consists of the theory of combinators or lambda calculus extended by extra constants (and corresponding axioms and rules) intended to capture inference. In a preceding paper, [2], we considered 4 systems of illative combinatory logic that are sound for first order intuitionistic prepositional and predicate logic. The interpretation from ordinary logic into the illative systems can be done in two ways: following the propositions-as-types paradigm, in which derivations become combinators, or in a more direct way, in which derivations are not translated. Both translations are closely related in a canonical way. In the cited paper we proved completeness of the two direct translations. In the present paper we prove that also the two indirect translations are complete. These proofs are direct whereas in another version, [3], we proved completeness by showing that the two corresponding illative systems are conservative over the two systems for the direct translations. Moreover we shall prove that one of the systems is also complete for predicate calculus with higher type functions.
\end{abstract}

\section{Keywords}

into, illative, interpretation, combinatory, intuitionistic, types, propositions, completeness, logic

Disciplines

Engineering | Science and Technology Studies

\section{Publication Details}

Dekkers, W., Bunder, M. \& Barendregt, H. (1998). Completeness of the propositions-as-types interpretation of intuitionistic logic into illative combinatory logic. The Journal of Symbolic Logic, 63 (3), 869-890. 


\title{
COMPLETENESS OF THE PROPOSITIONS-AS-TYPES INTERPRETATION OF INTUITIONISTIC LOGIC INTO ILLATIVE COMBINATORY LOGIC
}

\author{
WIL DEKKERS, MARTIN BUNDER, AND HENK BARENDREGT
}

\begin{abstract}
Illative combinatory logic consists of the theory of combinators or lambda calculus extended by extra constants (and corresponding axioms and rules) intended to capture inference. In a preceding paper, [2], we considered 4 systems of illative combinatory logic that are sound for first order intuitionistic propositional and predicate logic. The interpretation from ordinary logic into the illative systems can be done in two ways: following the propositions-as-types paradigm, in which derivations become combinators, or in a more direct way, in which derivations are not translated. Both translations are closely related in a canonical way. In the cited paper we proved completeness of the two direct translations. In the present paper we prove that also the two indirect translations are complete. These proofs are direct whereas in another version, [3], we proved completeness by showing that the two corresponding illative systems are conservative over the two systems for the direct translations. Moreover we shall prove that one of the systems is also complete for predicate calculus with higher type functions.
\end{abstract}

$\S 1$. Introduction. In [2] we introduced 4 systems of illative combinatory logic (ICL). We derived roughly the following soundness result

$$
\Delta \vdash_{\mathrm{L}} A \Rightarrow[\Delta] \vdash_{\mathrm{C}}[A],
$$

where $\mathrm{L}$ represents propositional or predicate logic and [-] one of two possible translations of each system into an ICL system C. Of the interpretations one is the propositions-as-types interpretation due to Curry, Howard and de Bruijn, the other is a more direct interpretation.

We derived completeness results for the direct translations of propositional and predicate calculus into 2 of the 4 systems of ICL. These, again roughly, took the following form

$$
[\Delta] \vdash_{\mathrm{C}}[A] \Rightarrow \Delta \vdash_{\mathrm{L}} A \text {. }
$$

In the present paper we shall prove that also the two indirect translations are complete. These completeness results imply the consistency of the ICL's involved. Moreover we shall prove that one of the systems is also complete for predicate calculus with higher type functions.

$\S 2$. Some definitions of preceding paper. This paper is a continuation of a preceding paper, [2], by the same authors. We will refer to that paper most of the time as B;

Received January 16, 1996; revised November 11, 1996.

Research supported by the Australian Research Council. 
so for example Definition B2.1 means Definition 2.1 in [2]. In this section we repeat some definitions of [2] that are needed in the present paper. For an introduction into ICL-systems, motivations, examples, propositions with proofs, and references we refer to [2].

Definition 2.1 (Definition B2.1; the logic system PROP). PROP is the $\supset$ fragment of intuitionistic propositional logic determined as follows.

(i) The set of formulas of PROP, notation $\mathbb{F}_{\mathrm{PROP}}$, is defined by the following abstract syntax

$$
\mathbb{F}_{\text {PROP }}=\mathbb{V} \mid \mathbb{F}_{\text {PROP }} \supset \mathbb{F}_{\text {PROP }}
$$

Here $\mathbb{V}$ is a set of propositional variables.

(ii) Let $\Gamma \subseteq \mathbb{F}_{\mathrm{PROP}}$ and $\varphi \in \mathbb{F}_{\mathrm{PROP}}$. Then $\Gamma \vdash_{\mathrm{PROP}} \varphi$ is defined by the following system of natural deduction.

$$
\begin{aligned}
& \text { PROP } \\
& \hline \varphi \in \Gamma \Rightarrow \Gamma \vdash \varphi ; \\
& \Gamma \vdash \varphi \supset \psi, \Gamma \vdash \varphi \Rightarrow \Gamma \vdash \psi ; \\
& \Gamma, \varphi \vdash \psi \Rightarrow \Gamma \vdash \varphi \supset \psi \\
& \hline
\end{aligned}
$$

DefinItIon 2.2 (Definition B2.2; the logic system PRED). PRED is the $\{\supset, \forall\}$ fragment of first order many-sorted intuitionistic predicate calculus of a given signature $s$.

Below as an example, we will treat a version of PRED with $s$ the signature of the structure

with

$$
\left\langle\mathbf{A}_{1}, \mathbf{A}_{2}, \mathbf{f}, \mathbf{g}, \mathbf{P}, \mathbf{a}\right\rangle
$$

$\mathbf{A}_{1}, \mathbf{A}_{2} \quad$ nonempty sets;

$\mathbf{f}: \mathbf{A}_{1} \rightarrow \mathbf{A}_{1}$ a unary function;

$\mathbf{g}: \mathbf{A}_{1} \rightarrow \mathbf{A}_{2} \rightarrow \mathbf{A}_{1} \quad$ a binary function;

$\mathbf{P} \subseteq \mathbf{A}_{1} \quad$ a unary relation;

$\mathbf{a} \in \mathbf{A}_{1}$ a constant.

(All results also hold for arbitrary signatures.)

(i) The set of terms of PRED, notation $\mathbb{T}_{\mathrm{PRED}}$, is defined by the following abstract syntax.

$$
\begin{aligned}
\mathbb{T}_{\mathrm{PRED}} & =\mathbb{T}_{\mathbf{A}_{1}} \mid \mathbb{T}_{\mathbf{A}_{2}} ; \\
\mathbb{T}_{\mathbf{A}_{1}} & =\mathbb{V}^{\mathbf{A}_{1}}|\mathbf{a}| \mathbf{f} \mathbb{T}_{\mathbf{A}_{1}} \mid \mathbf{g} \mathbb{T}_{\mathbf{A}_{1}} \mathbb{T}_{\mathbf{A}_{2}} ; \\
\mathbb{T}_{\mathbf{A}_{2}} & =\mathbb{V}^{\mathbf{A}_{2}} .
\end{aligned}
$$

(ii) The set of formulas of PRED, notation $\mathbb{F}_{\mathrm{PRED}}$, is defined by the following abstract syntax.

$$
\mathbb{F}_{\text {PRED }}=\mathbf{P} \mathbb{T}_{\mathbf{A}_{1}}\left|\mathbb{F}_{\text {PRED }} \supset \mathbb{F}_{\text {PRED }}\right| \forall \mathbb{V}^{\mathbf{A}_{i}} \mathbb{F}_{\text {PRED }} .
$$

(ii) $\Gamma \vdash_{\text {PRED }} \varphi$ is axiomatised by the following system of natural deduction. 
PRED

$\begin{aligned} \varphi \in \Gamma & \Rightarrow \Gamma \vdash \varphi ; \\ \Gamma \vdash \varphi \supset \psi, \Gamma \vdash \varphi & \Rightarrow \Gamma \vdash \psi ; \\ \Gamma, \varphi \vdash \psi & \Rightarrow \Gamma \vdash \varphi \supset \psi ; \\ \Gamma \vdash \forall x^{\mathbf{A}_{i}} \varphi, t \in \mathbb{T}_{\mathbf{A}_{i}} & \Rightarrow \Gamma \vdash \varphi\left[x^{\mathbf{A}_{i}}:=t\right] ; \\ \Gamma \vdash \varphi, \quad x^{\mathbf{A}_{i}} \notin \mathrm{FV}(\Gamma) & \Rightarrow \Gamma \vdash \forall x^{\mathbf{A}_{i}} \varphi .\end{aligned}$

The systems PROP and PRED are being interpreted in ICL's by two kinds of embeddings. The first kind is 'direct' and the second kind is according to the 'propositions-as-types' and 'proofs-as-terms' paradigm. As there are two logical systems, PROP and PRED, there are four systems of ICL. These systems are called $\mathscr{I} \mathbf{P}, \mathscr{I} \Xi, \mathscr{I} \mathbf{F}$, and $\mathscr{I} \mathbf{G}$ respectively. Their use for the two kinds of interpretation is as follows. Let []$^{1}$ be the direct, and [ $]^{2}$ the propositions-as-types translation. Then the following table shows the systems of ICL that are used for the two translations of PROP and PRED.

\begin{tabular}{c||c|c|} 
& {[]$^{1}$} & {[]$^{2}$} \\
\hline \hline PROP & $\mathscr{I P}$ & $\mathscr{I} \mathbf{F}$ \\
\hline PRED & $\mathscr{I} \Xi$ & $\mathscr{I} \mathbf{G}$ \\
\hline
\end{tabular}

For example

$$
[]^{2}: \mathrm{PRED} \rightarrow \mathscr{I} \mathbf{G}
$$

Now we will describe the four systems of ICL.

Definition 2.3 (Definition B2.3; the four systems of ICL). Let $T=\Lambda(\Xi, \mathbf{L})$, be the set of type-free lambda terms extended by the extra constants $\Xi$ and $\mathbf{L}$.

(i) Define the following terms in $\mathrm{T}$.

$$
\begin{aligned}
\mathbf{P} & \equiv \lambda x y . \Xi(\mathbf{K} x)(\mathbf{K} y), \\
\mathbf{F} & \equiv \lambda x y z . \Xi x(y \circ z), \\
\mathbf{G} & \equiv \lambda x y z . \Xi x(\mathbf{S} y z), \\
\mathbf{H} & \equiv \mathbf{L} \circ \mathbf{K},
\end{aligned}
$$

where $\mathbf{K} \equiv \lambda p q . p, \quad M \circ N \equiv \lambda x . M(N x)$ and $\mathbf{S} \equiv \lambda p q r . p r(q r)$.

Write $X \supset Y$ for $\mathbf{P} X Y$.

(ii) Define the following four systems of illative combinatory logic $\mathscr{I} \mathbf{P}, \mathscr{I} \Xi, \mathscr{I} \mathbf{F}$ and $\mathscr{I} \mathbf{G}$. $T$ is the set of terms of all 4 systems. A basis $\Gamma$ is a set of terms. All four systems have as rules the following:

$$
\begin{aligned}
& \text { All systems } \\
& \begin{aligned}
X \in \Gamma & \Rightarrow \Gamma \vdash X ; \\
\Gamma \vdash X, X={ }_{\beta \eta} Y & \Rightarrow \Gamma \vdash Y .
\end{aligned}
\end{aligned}
$$


The four systems have the following specific rules:

$\mathscr{I P}$
\begin{tabular}{|llll}
$\mathrm{P}_{\mathrm{e}}$ & $\Gamma \vdash X \supset Y, \Gamma \vdash X$ & $\Rightarrow \Gamma \vdash Y ;$ \\
$\mathrm{P}_{\mathrm{i}}$ & $\Gamma, X \vdash Y, \Gamma \vdash \mathbf{H} X$ & $\Rightarrow \Gamma \vdash X \supset Y ;$ \\
$\mathrm{P}_{\mathrm{H}}$ & $\Gamma, X \vdash \mathbf{H} Y, \Gamma \vdash \mathbf{H} X \Rightarrow \Gamma \vdash \mathbf{H}(X \supset Y)$. \\
\hline
\end{tabular}

\begin{tabular}{|llll|}
\multicolumn{1}{|l}{$\Xi$} & \\
\hline$\Xi_{\mathrm{e}}$ & $\Gamma \vdash \Xi X Y, \Gamma \vdash X V$ & $\Rightarrow$ & $\Gamma \vdash Y V ;$ \\
$\Xi_{\mathrm{i}}$ & $\Gamma, X x \vdash Y x, \Gamma \vdash \mathbf{L} X, \quad x \notin \mathrm{FV}(\Gamma, X, Y)$ & $\Rightarrow$ & $\Gamma \vdash \Xi X Y ;$ \\
$\Xi_{\mathrm{H}}$ & $\Gamma, X x \vdash \mathbf{H}(Y x), \Gamma \vdash \mathbf{L} X, \quad x \notin \mathrm{FV}(\Gamma, X, Y)$ & $\Rightarrow \Gamma \vdash \mathbf{H}(\Xi X Y)$.
\end{tabular}

$\mathscr{I} \mathbf{F}$

\begin{tabular}{|llll}
\hline $\mathrm{F}_{\mathrm{e}}$ & $\Gamma \vdash \mathbf{F} X Y Z, \quad \Gamma \vdash X V$ & $\Rightarrow \Gamma \vdash Y(Z V) ;$ \\
$\mathrm{F}_{\mathrm{i}}$ & $\Gamma, X x \vdash Y(Z x), \quad \Gamma \vdash \mathbf{L} X, \quad x \notin \mathrm{FV}(\Gamma, X, Y, Z)$ & $\Rightarrow \Gamma \vdash \mathbf{F} X Y Z ;$ \\
$\mathrm{F}_{\mathrm{L}}$ & $\Gamma, X x \vdash \mathbf{L} Y, \quad \Gamma \vdash \mathbf{L} X, \quad x \notin \mathrm{FV}(\Gamma, X, Y)$ & $\Rightarrow \Gamma \vdash \mathbf{L}(\mathbf{F} X Y)$.
\end{tabular}

$\mathscr{I} \mathbf{G}$

\begin{tabular}{lllll}
\hline $\mathrm{G}_{\mathrm{e}}$ & $\Gamma \vdash \mathbf{G} X Y Z, \Gamma \vdash X V$ & $\Rightarrow$ & $\Gamma \vdash Y V(Z V) ;$ \\
$\mathrm{G}_{\mathrm{i}}$ & $\Gamma, X x \vdash Y x(Z x), \quad \Gamma \vdash \mathbf{L} X, \quad x \notin \mathrm{FV}(\Gamma, X, Y, Z)$ & $\Rightarrow$ & $\Gamma \vdash \mathbf{G} X Y Z ;$ \\
$\mathrm{G}_{\mathrm{L}}$ & $\Gamma, X x \vdash \mathbf{L}(Y x), \quad \Gamma \vdash \mathbf{L} X, \quad x \notin \mathrm{FV}(\Gamma, X, Y)$ & $\Rightarrow$ & $\Gamma \vdash \mathbf{L}(\mathbf{G} X Y)$.
\end{tabular}

DefINITION 2.4 (Definition B2.8; interpretation of PROP). Let $r$ be a closed term in $\Lambda(\Xi, \mathbf{L})$. Two maps (for $i=1,2$ )

$$
[-]_{r}^{i}: \mathbb{F}_{\mathrm{PROP}} \rightarrow \Lambda(\Xi, \mathbf{L})
$$

and two maps

$$
\Gamma_{r}^{i}: \mathbb{F}_{\mathrm{PROP}} \rightarrow \text { illative contexts }
$$

are defined by the following table. (Note that these illative contexts are effectively grammatical conditions on the variables (propositional, individual) that appear in a proposition.)

\begin{tabular}{|c||c|c||c|c|}
\hline$\varphi$ & {$[\varphi]_{r}^{1}$} & $\Gamma_{r}^{1}(\varphi)$ & {$[\varphi]_{r}^{2}$} & $\Gamma_{r}^{2}(\varphi)$ \\
\hline$p$ & $r p$ & $\mathbf{H}(r p)$ & $r p$ & $\mathbf{L}(r p)$ \\
$\psi \supset \chi$ & {$[\psi]_{r}^{1} \supset[\chi]_{r}^{1}$} & $\Gamma_{r}^{1}(\psi), \Gamma_{r}^{1}(\chi)$ & $\mathbf{F}[\psi]_{r}^{2}[\chi]_{r}^{2}$ & $\Gamma_{r}^{2}(\psi), \Gamma_{r}^{2}(\chi)$ \\
\hline
\end{tabular}

REMARK 2.5. The $r$ in the above can be replaced by I (i.e., omitted). In other versions of this paper however we specialised to $r=\mathbf{K}$. (cf., Remark 7.1.) Moreover in Proposition B2.15 we used it to derive a relation between the two interpretations. 
DeFINITION 2.6 (Definition B2.12; interpretation of PRED).

(i) $\Lambda^{s}(\Xi, \mathbf{L})$ is $\Lambda(\Xi, \mathbf{L})$ extended by the extra constants $A_{1}, A_{2}, P, f, g, a$ associated with the signature $s$ of the manysorted structure of our example. Because we are going to interprete many sorted predicate logic with sorts $\mathbf{A}_{1}, \mathbf{A}_{2}$, it is useful to have among the free variables of the $\lambda$-calculus infinite sets $\mathscr{V}_{1}, \mathscr{V}_{2}$, with $\mathscr{V}_{i}=\left\{x_{i}, y_{i}, z_{i} \ldots\right\} . x, y, z$ denote arbitrary variables.

(ii) Let $r$ be a closed term in $\Lambda^{s}(\Xi, \mathbf{L})$. Two maps (for $i=1,2$ )

$$
[-]_{r}^{i}: \mathbb{F}_{\mathrm{PRED}} \rightarrow \Lambda^{s}(\Xi, \mathbf{L})
$$

and a map

$$
\Gamma: \mathbb{F}_{\text {PRED }} \rightarrow \text { illative contexts }
$$

are defined by the following tables:

\begin{tabular}{|c|c|c|}
\hline$t$ & {$[t]_{r}^{i}$} & $\Gamma(t)$ \\
\hline$x^{\mathbf{A}_{j}}$ & $x_{j}$ & $A_{j} x_{j}$ \\
$\mathbf{a}$ & $a$ & $\emptyset$ \\
$\mathbf{f} s$ & $f[s]_{r}^{i}$ & $\Gamma(s)$ \\
$\mathbf{g} s t$ & $g[s]_{r}^{i}[t]_{r}^{i}$ & $\Gamma(s), \Gamma(t)$ \\
\hline
\end{tabular}

\begin{tabular}{|c||c|c|c|}
\hline$\varphi$ & {$[\varphi]_{r}^{1}$} & {$[\varphi]_{r}^{2}$} & $\Gamma(\varphi)$ \\
\hline $\mathbf{P} t$ & $r\left(P[t]_{r}^{1}\right)$ & $r\left(P[t]_{r}^{2}\right)$ & $\Gamma(t)$ \\
$\psi \supset \chi$ & {$[\psi]_{r}^{1} \supset[\chi]_{r}^{1}$} & $\mathbf{F}[\psi]_{r}^{2}[\chi]_{r}^{2}$ & $\Gamma(\psi), \Gamma(\chi)$ \\
$\forall x^{\mathbf{A}_{i}} \psi$ & $\Xi A_{i}\left(\lambda x_{i} \cdot[\psi]_{r}^{1}\right)$ & $\mathbf{G} A_{i}\left(\lambda x_{i} \cdot[\psi]_{r}^{2}\right)$ & $\Gamma(\psi)-\left\{A_{i} x_{i}\right\}$ \\
\hline
\end{tabular}

(iii)

$$
\begin{aligned}
\Gamma_{r, s}^{1} & =\left\langle\mathbf{L} A_{1}, \mathbf{L} A_{2}, \mathbf{F} A_{1} A_{1} f, \mathbf{F} A_{1}\left(\mathbf{F} A_{2} A_{1}\right) g, \mathbf{F} A_{1} \mathbf{H}(r \circ P), A_{1} a\right\rangle, \\
\Gamma_{r, s}^{2} & =\left\langle\mathbf{L} A_{1}, \mathbf{L} A_{2}, \mathbf{F} A_{1} A_{1} f, \mathbf{F} A_{1}\left(\mathbf{F} A_{2} A_{1}\right) g, \mathbf{F} A_{1} \mathbf{L}(r \circ P), A_{1} a\right\rangle
\end{aligned}
$$

and

$$
\Gamma_{r, s}^{i,+}=\Gamma_{r, s}^{i} \cup\left\{A_{2} x_{2}\right\} \text { where } x_{2} \in \mathscr{V}_{2} \text { is some variable. }
$$

The definitions $\Gamma_{r, s}^{i}$ and $\Gamma_{r, s}^{i,+}$ of course refer to our example of a many-sorted predicate calculus with signature $s$.

§3. Summary of results in preceding and present paper. We had as aim in the preceding paper to prove

(i) $\Delta \vdash_{\text {PROP }} \varphi \Leftrightarrow \forall$ closed $r\left[\cdots 1,[\Delta]_{r}^{1} \vdash \mathscr{I P}_{\mathrm{P}}[\varphi]_{r}^{1}\right]$;

(ii) $\Delta \vdash_{\operatorname{PRED}} \varphi \Leftrightarrow \forall$ closed $r\left[\cdots 1,[\Delta]_{r}^{1} \vdash_{\mathscr{G} \Xi}[\varphi]_{r}^{1}\right]$;

(iii) $\Delta \vdash_{\mathrm{PROP}} \varphi \Leftrightarrow \forall$ closed $r \exists M\left[\ldots 2,[\Delta]_{r}^{2} \vdash_{\mathscr{I} \mathrm{F}}[\varphi]_{r}^{2} M\right]$;

(iv) $\Delta \vdash_{\mathrm{PRED}} \varphi \Leftrightarrow \forall$ closed $r \exists M\left[\ldots 2,[\Delta]_{r}^{2} \vdash_{\mathscr{I}_{\mathrm{G}}}[\varphi]_{r}^{2} M\right]$;

where $\ldots_{i}$ stands for $\Gamma_{r}^{i}(\Delta, \varphi)$ in the cases of $\operatorname{PROP}$ and for $\Gamma_{r, s}^{i,+}, \Gamma(\Delta, \varphi)$ in the cases of PRED.

The proofs of soundness $(\Rightarrow)$ were easy. (cf., Propositions B2.11 and B2.14). In Propositions B3.14 and B3.11 we proved completeness for the 2 direct translations []$^{1}: \mathrm{PROP} \rightarrow \mathscr{I P}$ and []$^{1}: \mathrm{PRED} \rightarrow \mathscr{I} \Xi,((\mathrm{i}) \Leftarrow$ and $(\mathrm{ii}) \Leftarrow)$, by specialising 
to $r=\mathbf{I}$. In the present paper we prove completeness for the 2 indirect translations []$^{2}:$ PROP $\rightarrow \mathscr{I F}$ and []$^{2}:$ PRED $\rightarrow \mathscr{I} \mathbf{G},(($ iii $) \Leftarrow$ and $($ iv $) \Leftarrow)$, also by specialising to $r=I$. In Section 7 we point out that completeness for all 4 cases can be proved also by specialising to $r=\mathbf{K}$.

The completeness proof for the direct translation [ ] $]^{1}:$ PRED $\rightarrow \mathscr{I} \Xi((i i) \Leftarrow)$ was given in the following way. We had to show

$$
\Gamma_{s}^{1,+},[\Delta]^{1}, \Gamma(\Delta, \varphi) \vdash_{\mathscr{S} \Xi}[\varphi]^{1} \Rightarrow \Delta \vdash_{\mathrm{PRED}} \varphi .
$$

(cf., page B781.) Write $\Gamma^{\prime}=\Gamma_{s}^{1,+},[\Delta]^{1}, \Gamma(\Delta, \varphi)$. First we defined in B3.2 a set $\mathscr{O}$ in order to analyze the terms $M$ such that $\Gamma^{\prime} \vdash \mathscr{g} \Xi M$. We defined $\overline{\mathscr{O}}=\{M|\exists N \in \mathscr{O}|$ $\left.N={ }_{\beta \eta} M\right\}$ and it was clear that $\Gamma^{\prime} \subseteq \overline{\mathscr{O}}$. We showed in Proposition B3.10 that $\mathscr{O}$ is an invariant (modulo $\beta \eta$-equality) for derivations in $\mathscr{I} \Xi$ :

$$
\Gamma \vdash \mathscr{g} \Xi M, \Gamma \subseteq \overline{\mathscr{O}} \Rightarrow M \in \overline{\mathscr{O}} .
$$

Using this result we then proved in Proposition B3.11

$$
\Gamma^{\prime} \vdash \mathscr{g} \Xi[\varphi]^{1} \Rightarrow \Delta \vdash_{\mathrm{PRED}} \varphi .
$$

The completeness proof for []$^{1}:$ PROP $\rightarrow \mathscr{I P}((\mathrm{i}) \Leftarrow)$ was done in a similar but easier way.

In the present paper we will prove completeness of []$^{2}:$ PROP $\rightarrow \mathscr{I F}$ and []$^{2}:$ PRED $\rightarrow \mathscr{I} \mathbf{G}((\mathrm{iii}) \Leftarrow$ and $(\mathrm{iv}) \Leftarrow)$.

We start with the most difficult case, the proof of completeness of []$^{2}:$ PRED $\rightarrow$ $\mathscr{I} \mathbf{G}$. We define in Definition 4.2 a set $\mathscr{O}_{G}$ and we will show in Proposition 4.10 that $\mathscr{O}_{G}$ is an invariant (modulo $\beta \eta$-equality) for derivations in $\mathscr{I} \mathbf{G}:$

$$
\Gamma \vdash_{\mathcal{F}_{\mathrm{G}}} M, \Gamma \subseteq \overline{\mathscr{O}_{G}} \Rightarrow M \in \overline{\mathscr{O}_{G}} .
$$

The invariant $\mathscr{O}_{G}$ is more complicated than $\mathscr{O}$ of B3.2 and we cannot use it to prove completeness directly in a way similar to the proof of completeness for $\mathscr{I} \Xi$ relative to PRED by means of $\mathscr{O}$ in B3.11. In fact we shall use $\mathscr{O}_{G}$ in order to show in Lemma 4.18 that in a proof of $\Gamma \vdash_{\mathscr{I}_{\mathrm{G}}} M$, where $\Gamma \subseteq \overline{\mathscr{O}}_{G}$, all cut formulas can be eliminated. Then we define in Definition 4.21 a second invariant $\mathscr{O}_{1}$. This $\mathscr{O}_{1}$ plays a role similar to that of $\mathscr{O}$ in B3.2. Using this invariant we prove in $4.22-4.25$ the completeness in a way similar to the proof of completeness of []$^{1}:$ PRED $\rightarrow \mathscr{I} \Xi$ in B3.2-B3.11.

Completenes of $\mathscr{I} \mathbf{F}$ for PROP is easier. There is no need for cut elimination and we give the proof directly, using an invariant $\mathscr{O}_{2}$ similar to $\mathscr{O}$ and $\mathscr{O}_{1}$.

In the last part of the paper we shall introduce another logic system: Predicate Calculus with Higher Type Functions, notation PRED $\lambda \rightarrow$. We shall prove in Proposition 6.12 that $\mathscr{I} \mathbf{G}$ is also complete for PRED $\lambda \rightarrow$ under the second translation []$^{2}$.

§4. Completeness of $\mathscr{I} \mathbf{G}$ for PRED. We will show for $\varphi \in \mathbb{F}_{\mathrm{PRED}}, \Delta \subseteq \mathbb{F}_{\mathrm{PRED}}$ : $\forall$ closed $r \exists M\left[\Gamma_{r, s}^{2,+},[\Delta]_{r}^{2}, \Gamma(\Delta, \varphi) \vdash_{\mathscr{I}_{\mathrm{G}}}[\varphi]_{r}^{2} M\right] \Rightarrow \Delta \vdash_{\mathrm{PRED}} \varphi$.

It is sufficient to show

$$
\Gamma_{\mathbf{l}, s}^{2,+},[\Delta]_{\mathbf{l}}^{2}, \Gamma(\Delta, \varphi) \vdash_{\mathscr{I}_{\mathrm{G}}}[\varphi]_{\mathbf{1}}^{2} M \Rightarrow \Delta \vdash_{\mathrm{PRED}} \varphi .
$$


We omit the index I in the notation and we prove

$$
\Gamma_{s}^{2,+},[\Delta]^{2}, \Gamma(\Delta, \varphi) \vdash_{\mathscr{I}_{\mathrm{G}}}[\varphi]^{2} M \Rightarrow \Delta \vdash_{\mathrm{PRED}} \varphi .
$$

The proof is again in several steps. First we define in Definition 4.2 an invariant $\mathscr{O}_{G}$ (modulo $\beta \eta$-equality) for derivations in $\mathscr{I} \mathbf{G}$. Using this invariant we show in Lemma 4.18 that in a proof of $\Gamma_{s}^{2,+},[\Delta]^{2}, \Gamma(\Delta, \varphi) \vdash_{\mathscr{A}_{\mathrm{G}}} N$ all cuts can be eliminated. Then we define in Definition 4.21 a second invariant $\mathscr{O}_{1}$, similar to $\mathscr{O}$ in B3.2 and using this invariant we prove in $4.22-4.25$ the completeness in a way similar to the proof of completeness of []$^{1}: \mathrm{PRED} \rightarrow \mathscr{I} \Xi$ in B3.2 - B3.11. Instead of $\vdash_{\mathscr{I}_{\mathrm{G}}}$ we shall mostly write $\vdash$.

Now we repeat Definition B3.2 of the invariant $\mathscr{O}$ for derivations in $\mathscr{I} \Xi$ for the manysorted structure of our example, in order to contrast it with the one for derivations in $\mathscr{I} \mathbf{G}$ of Definition 4.2 and also because we shall refer to it often in the sequel. Instead of $x_{i}, \mathscr{V}_{i}$ and $\mathscr{T}_{i}$ we shall write $x^{A_{i}}, \mathscr{V}^{A_{i}}$ and $\mathscr{T}^{A_{i}}$.

Definition 4.1 (Invariant for derivations in $\mathscr{I} \Xi$ ).

$$
\begin{aligned}
\mathscr{T} & \equiv \mathscr{T}^{A_{1}} \mid \mathscr{T}^{A_{2}} ; \\
\mathscr{T}^{A_{1}} & \equiv \mathscr{V}^{A_{1}}|a| f \mathscr{T}^{A_{1}} \mid g \mathscr{T}^{A_{1}} \mathscr{T}^{A_{2}} ; \\
\mathscr{T}^{A_{2}} & \equiv \mathscr{V}^{A_{2}} ; \\
\mathscr{P} & \equiv P \mathscr{T}^{A_{1}}\left|\Xi A_{i}\left(\lambda x^{A_{i}} \cdot \mathscr{P}\right)\right| \Xi(\mathbf{K} \mathscr{P})(\mathbf{K} \mathscr{P}) ; \\
\mathscr{G} & \equiv \mathbf{L} A_{i}\left|A_{i} \mathscr{T}^{A_{i}}\right| \Xi A_{i}\left(\lambda x^{A_{i}} \cdot \mathscr{G}\right)|\Xi(\mathbf{K} \mathscr{P})(\mathbf{K} \mathscr{G})| \mathbf{L}(\mathbf{K} \mathscr{P}) ; \\
\mathscr{O} & \equiv \mathscr{G} \mid \mathscr{P} .
\end{aligned}
$$

Now we will define the invariant $\mathscr{O}_{G}$ for derivations in $\mathscr{I} \mathbf{G}$. $\mathscr{O}_{G}$ is more complicated then the invariant $\mathscr{O}$ for $\mathscr{I} \Xi$ for several reasons. If $\Gamma_{s}^{1,+},[\Delta]^{1}, \Gamma(\Delta, \varphi) \vdash \mathbf{L} X$ in system $\mathscr{I} \Xi$ then $X=A_{i}$ or $X=\mathbf{K} p$ with $p \in \mathscr{P}$. But if $\Gamma_{s}^{2,+},[\Delta]^{2}, \Gamma(\Delta, \varphi) \vdash \mathbf{L} X$ in system $\mathscr{I} \mathbf{G}$ then we can have for example $X=\mathbf{F} A_{1} A_{2}$ or even $X=\mathbf{F} p A_{2}$. Therefore we define an extension $\mathscr{L}_{G}$ of the set of sorts $\left\{A_{1}, A_{2}\right\}$ containing these $X$ 's. We need for each $l \in \mathscr{L}_{G}$ a set of variables $\mathscr{V}_{G}^{l}$, similar to the sets $\mathscr{V}^{A_{1}}$ and $\mathscr{V}^{A_{2}}$. If we took a different set of variables for each $l \in \mathscr{L}_{G}$, then the Substitution Lemma 4.6, that is needed in the Proof of Proposition 4.10, would not hold. Therefore we define for each $l \in \mathscr{L}_{G}$ the skeleton $s k(l)$, such that $s k(p)$ is the same for all $p \in \mathscr{P}_{G}$ and we define $\mathscr{V}_{G}^{l_{1}}=\mathscr{V}_{G}^{l_{2}}$ iff $s k\left(l_{1}\right)=s k\left(l_{2}\right)$.

The sets $\mathscr{T}_{G}^{l}$ will contain $\lambda$-abstractions: if $l \equiv \mathbf{F} l_{1} l_{2}$ and $t^{l_{2}} \in \mathscr{T}_{G}^{l_{2}}$ then $\lambda x^{l_{1}} \cdot t^{l_{2}} \in \mathscr{T}_{G}^{l}$. This may seem a little bit strange, but in fact it is very natural. For example we have $\vdash \mathbf{F} A_{1} A_{1}\left(\lambda x^{A_{1}} \cdot x^{A_{1}}\right)$ by rule $\mathrm{F}_{\mathrm{i}}$. Hence $\lambda x^{A_{1}} \cdot x^{A_{1}}$ should be in $\mathscr{T}_{G}^{\mathbf{F}_{1} A_{1}}$. Moreover in the Proof of Lemma 4.9 we really need that $\lambda x^{l_{1}} \cdot t^{l_{2}} \in \mathscr{T}_{G}^{\mathrm{F} l_{1} l_{2}}$.

Finally we had $\Xi(\mathbf{K} p)(\mathbf{K} O) \in \mathscr{O}$ whereas now we have $\Xi p\left(\lambda x^{p} . O\right) \in \mathscr{O}_{G}$, where $x^{p}$ may occur free in $O$.

Definition 4.2 (Invariant $\mathscr{O}_{G}$ for derivations in $\mathscr{F} \mathbf{G}$ ). (i) $\Lambda^{s}(\Xi, \mathbf{L})$ is, as in Definition $\mathrm{B} 2.12, \Lambda(\Xi, \mathrm{L})$ extended by the extra constants $A_{1}, A_{2}, P, f, g, a$ associated with the signature $s$ of the manysorted structure of our example.

(ii) Now we will define simultaneously a set $\mathscr{L}_{G}$ of sorts (an extension of the set $\left.\left\{A_{1}, A_{2}\right\}\right)$, for each $l \in \mathscr{L}_{G}$ the skeleton $s k(l)$, a set of variables $\mathscr{V}_{G}^{l}$ and a set of terms $\mathscr{T}_{G}^{l}$, sets $\mathscr{P}_{G}, \mathscr{H}_{G}, \mathscr{G}_{G}$ and $\mathscr{O}_{G}$. 
Definition of $\mathscr{L}_{G}$.

$$
\mathscr{L}_{G} \equiv A_{1}\left|A_{2}\right| P t^{A_{1}} \mid \mathbf{G} l_{1}\left(\lambda x^{l_{1}} . l_{2}\right) \quad\left(t^{A_{1}} \in \mathscr{T}_{G}^{A_{1}}, l_{1}, l_{2} \in \mathscr{L}_{G}, x^{l_{1}} \in \mathscr{V}_{G}^{l_{1}}\right) .
$$

Definition of $\operatorname{sk}(l)$.

$$
\begin{aligned}
s k\left(A_{i}\right) & \equiv A_{i} ; \\
s k\left(P t^{A_{1}}\right) & \equiv P x^{A_{1}} \quad\left(\text { i.e., } s k\left(P t^{A_{1}}\right) \text { is independent of } t^{A_{1}}\right) ; \\
s k\left(\mathbf{G} l_{1}\left(\lambda x^{l_{1}} \cdot l_{2}\right)\right) & \equiv \mathbf{G}\left(\operatorname{sk}\left(l_{1}\right)\right)\left(\lambda x^{l_{1}} \cdot s k\left(l_{2}\right)\right) .
\end{aligned}
$$

Definition of $\mathscr{V}_{G}^{l}$.

We assume that for each skeleton $s k(l)$ there is a set of variables

$$
\mathscr{V}_{G}^{s k(l)} \equiv\left\{x^{s k(l)}, x_{1}^{s k(l)}, x_{2}^{s k(l)}, \ldots y^{s k(l)}, y_{1}^{s k(l)}, y_{2}^{s k(l)}, \ldots\right\}
$$

such that $\mathscr{V}_{G}^{s k\left(l_{1}\right)} \cap \mathscr{V}_{G}^{s k\left(l_{2}\right)}=\emptyset$ if $s k\left(l_{1}\right) \not \equiv s k\left(l_{2}\right)$, and for each $l \in \mathscr{L}_{G}$ we define

$$
\mathscr{V}_{G}^{l} \equiv \mathscr{V}_{G}^{s k(l)}, \quad x^{l} \equiv x^{s k(l)}, x_{i}^{l} \equiv x_{i}^{s k(l)}, \quad y^{l} \equiv y^{s k(l)}, y_{i}^{l} \equiv y_{i}^{s k(l)} .
$$

Definition of $\mathscr{T}_{G}^{l}$.

$$
\begin{array}{rlrl}
\mathscr{T}_{G}^{l} \equiv a\left|\mathscr{V}_{G}^{l}\right| \mathscr{T}_{G}^{\mathrm{G} l_{1}\left(\lambda x^{l_{1}} . l\right)} \mathscr{T}_{G}^{l_{1}}, & \text { if } l \equiv A_{1} ; \\
\mathscr{T}_{G}^{l} \equiv \mathscr{V}_{G}^{l} \mid \mathscr{T}_{G}^{\mathrm{G} l_{1}\left(\lambda x^{l_{1}} . l\right)} \mathscr{T}_{G}^{l_{1}}, & \text { if } l \equiv A_{2} \text { or } l \equiv P t^{A_{1}} ; \\
\mathscr{T}_{G}^{l} \equiv f\left|\mathscr{V}_{G}^{l}\right| \mathscr{T}_{G}^{\mathrm{G} l_{1}\left(\lambda x^{l_{1}} . l\right)} \mathscr{T}_{G}^{l_{1}} \mid \lambda x^{A_{1}} \cdot \mathscr{T}_{G}^{A_{1}}, & & \text { if } l \equiv \mathbf{F} A_{1} A_{1} ; \\
\mathscr{T}_{G}^{l} \equiv g\left|\mathscr{V}_{G}^{l}\right| \mathscr{T}_{G}^{\mathrm{G} l_{1}\left(\lambda x^{l_{1}} . l\right)} \mathscr{T}_{G}^{l_{1}} \mid \lambda x^{A_{1}} . \mathscr{T}_{G}^{\mathrm{F} A_{2} A_{1}}, & \text { if } l \equiv \mathbf{F} A_{1}\left(\mathbf{F} A_{2} A_{1}\right) ; \\
\mathscr{T}_{G}^{l} \equiv & \mathscr{V}_{G}^{l}\left|\mathscr{T}_{G}^{\mathrm{G} l_{1}\left(\lambda x^{l_{1}} . l\right)} \mathscr{T}_{G}^{l_{1}}\right| \lambda x^{l_{2}} . \mathscr{T}_{G}^{l_{3}}, & & \text { if } l \equiv \mathbf{G} l_{2}\left(\lambda x^{l_{2}} \cdot l_{3}\right), \\
& & l \notin\left\{\mathbf{F} A_{1} A_{1}, \mathbf{F} A_{1}\left(\mathbf{F} A_{2} A_{1}\right)\right\} .
\end{array}
$$

Definition of $\mathscr{P}_{G}, \mathscr{H}_{G}, \mathscr{G}_{G}$ and $\mathscr{O}_{G}$.

$$
\begin{aligned}
\mathscr{P}_{G} & \equiv P \mathscr{T}_{G}^{A_{1}} \mid \mathbf{G} l\left(\lambda x^{l} \cdot \mathscr{P}_{G}\right) ; \\
\mathscr{H}_{G} & \equiv\left(P t^{A_{1}}\right) t^{P t^{A_{1}}} \mid \Xi l\left(\lambda x^{l} \cdot \mathscr{H}_{G}\right) ; \\
\mathscr{G}_{G} & \equiv \mathbf{L} \mathscr{L}_{G}\left|A_{i} \mathscr{T}_{G}^{A_{i}}\right| \Xi l\left(\lambda x^{l} \cdot \mathscr{G}_{G}\right) ; \\
\mathscr{O}_{G} & \equiv \mathscr{H}_{G} \mid \mathscr{G}_{G} .
\end{aligned}
$$

(iii) Notation.

$$
\begin{aligned}
& l, l_{1}, l_{2}, l^{\prime}, \ldots \text { denote elements of } \mathscr{L}_{G} ; \\
& p, p_{1}, p_{2}, p^{\prime}, \ldots \text { denote elements of } \mathscr{P}_{G} .
\end{aligned}
$$

REMARKs 4.3. (i) If $\mathrm{FV}\left(l_{2}\right)=\emptyset$ then $\mathbf{G} l_{1}\left(\lambda x^{l_{1}} \cdot l_{2}\right)=_{\beta} \mathbf{F} l_{1} l_{2}$. In that case we will denote $\mathbf{G} l_{1}\left(\lambda x^{l_{1}} . l_{2}\right)$ usually by $\mathbf{F} l_{1} l_{2}$. In fact we did that already in the above definition.

(ii) The grammar in the definition of $\mathscr{O}_{G}$ is not context-free and this causes some inaccuracies in the notation. In the definition of $\mathscr{P}_{G}$ (and similarly at some other places) instead of $\mathbf{G l}\left(\lambda x^{l} \cdot \mathscr{P}_{G}\right)$ we should write $\mathbf{G} \mathscr{L}_{G}\left(\lambda x^{\mathscr{L}_{G}} \cdot \mathscr{P}_{G}\right)$, but then we do not know that these two $\mathscr{L}_{G}$ 's represent the same $l \in \mathscr{L}_{G}$. (We may not have $\left.\mathbf{G} l_{1}\left(\lambda x^{l_{2}} \cdot \mathscr{P}_{G}\right).\right)$ 
(iii) In the definition of $\mathscr{T}_{G}^{l}$ instead of the clause $\mathscr{T}_{G}^{\mathrm{G} l_{1}\left(\lambda x^{l_{1}} . l\right)} \mathscr{T}_{G}^{l_{1}}$ one might expect, in view of rule $\mathrm{G}_{\mathrm{e}}$ a clause $t_{1}^{\mathrm{G} l_{1}\left(\lambda x^{l_{1}} . l^{\prime}\right)} t_{2}^{l_{1}}$ where $l=l^{\prime}\left[x^{l_{1}}:=t_{2}^{l_{1}}\right]$, but that does not make a difference because we have for $l \in \mathscr{L}_{G}$

$$
\mathscr{T}_{G}^{l\left[x^{l_{1}}:=t^{l_{1}}\right]}=\mathscr{T}_{G}^{l},
$$

as follows easily from

$$
\mathscr{V}_{G}^{l\left[x^{l_{1}}:=t^{l_{1}}\right]}=\mathscr{V}_{G}^{l}
$$

in Lemma 4.6.

LEMMA 4.4. (i) $\Xi l\left(\lambda x^{l} \cdot p t\right)=\mathbf{G} l\left(\lambda x^{l} \cdot p\right)\left(\lambda x^{l} \cdot t\right)$;

(ii) $\mathbf{G} l\left(\lambda x^{l} \cdot p\right) t=\Xi l\left(\lambda x^{l} \cdot p\left(t x^{l}\right)\right), \quad x^{l} \notin \mathrm{FV}(t)$;

(iii) $\Xi l\left(\lambda x^{l} \cdot p t^{p}\right)=\mathbf{G} l\left(\lambda x^{l} \cdot p\right) t^{\mathbf{G} l\left(\lambda x^{l} \cdot p\right)}, \quad$ for $t^{\mathbf{G} l\left(\lambda x^{l} \cdot p\right)}=\lambda x^{l} \cdot t^{p}$;

(iv) $\mathbf{G} l\left(\lambda x^{l} \cdot p\right) t^{\mathbf{G} l\left(\lambda x^{l} \cdot p\right)}=\Xi l\left(\lambda x^{l} \cdot p t^{p}\right), \quad$ for $t^{p}=t^{\mathbf{G} l\left(\lambda x^{l} \cdot p\right)} x^{l}$;

(v) $\mathscr{H}_{G}=\mathscr{P}_{G} \mathscr{T}_{G}^{\mathscr{P}_{G}}$ modulo $\beta \eta$-equality.

Proof. (i), (ii) immediately from the definition of $\mathbf{G}$;

(iii), (iv) from (i), (ii);

(v) from (iii) and (iv).

REMARK 4.5. By (v) in the previous lemma we have $\mathscr{H}_{G}=\mathscr{P}_{G} \mathscr{T}_{G}^{\mathscr{P}_{G}}$. In B3.2 we defined $\mathscr{O}=\mathscr{P} \mid \mathscr{G}$ whereas now we have $\mathscr{O}_{G}=\mathscr{H}_{G} \mid \mathscr{G}_{G}$. This corresponds with the fact that now we treat derivations $\Gamma \vdash[\varphi]^{2} M$ instead of $\Gamma \vdash[\varphi]^{1}$.

LeMmA 4.6. Let $\mathscr{W}=\mathscr{L}_{G}, \mathscr{T}_{G}^{l}, \mathscr{P}_{G}, \mathscr{H}_{G}, \mathscr{G}_{G}$, or $\mathscr{O}_{G}$ and let $w \in \mathscr{W}, x^{l_{1}} \in \mathscr{V}_{G}^{l_{1}}$,

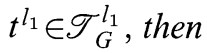

$$
w\left[x^{l_{1}}:=t^{l_{1}}\right] \in \mathscr{W},
$$

moreover if $\mathscr{W}=\mathscr{L}_{G}$ then

$$
\operatorname{sk}\left(w\left[x^{l_{1}}:=t^{l_{1}}\right]\right) \equiv \operatorname{sk}(w),
$$

and hence

$$
\mathscr{V}_{G}^{w\left[x^{l_{1}}:=t^{l_{1}}\right]} \equiv \mathscr{V}_{G}^{w}
$$

Proof. By induction on the structure of $w$. Instead of $x^{l_{1}}$ and $t^{l_{1}}$ we shall write $x$ and $t$.

CASE $w \in \mathscr{L}_{G}$.

$w \equiv A_{i}$. This is trivally ok.

$w \equiv P t_{1}^{A_{1}}$. This follows from the case $w \equiv t_{1}^{A_{1}}$.

$$
\begin{gathered}
w \equiv \mathbf{G} l_{2}\left(\lambda y^{l_{2}} \cdot l_{3}\right) \text {. Then } w[x:=t] \equiv \mathbf{G}\left(l_{2}[x:=t]\right)\left(\lambda y^{l_{2}} \cdot l_{3}[x:=t]\right) \equiv \\
\\
\equiv \mathbf{G}\left(l_{2}[x:=t]\right)\left(\lambda y^{l_{2}[x:=t]} \cdot l_{3}[x:=t]\right)
\end{gathered}
$$

(by the second part of the IH for $l_{2}$ ) and this is in $\mathscr{L}_{G}$ by the first part of the $\mathrm{IH}$ for $l_{2}$ and $l_{3}$. The second part of the IH for $l_{2}$ and $l_{3}$ yields $s k(w[x:=t]) \equiv s k(w)$. 
CASE $w=t^{l} \in \mathscr{T}_{G}^{l}$.

$l \equiv A_{1}$. Now $w \equiv x_{1}^{A_{1}}, a$ or $t_{1}^{G l_{1}\left(\lambda x_{1}^{l_{1}} \cdot A_{1}\right)} t_{2}^{l_{1}}$. All these cases are easy.

$l \equiv A_{2}$ or $P t_{1}^{A_{1}}$. Easy.

$l \equiv \mathbf{G} l_{2}\left(\lambda y^{l_{2}} \cdot l_{3}\right)$. Now $w \equiv x_{1}^{l}, t_{1}^{\mathbf{G} l_{4}\left(\lambda x_{1}^{l_{4}} \cdot l\right)} t_{2}^{l_{4}}, \lambda y^{l_{2}} \cdot t_{1}^{l_{3}}, f$ or $g$. Easy again.

CASE $w \in \mathscr{P}_{G}$.

$w \equiv P t_{1}^{A_{1}}$. This follows from the case $w \equiv t_{1}^{A_{1}}$.

$w \equiv \mathbf{G} l_{2}\left(\lambda y^{l_{2}} \cdot p\right)$. Then $w[x:=t] \equiv \mathbf{G}\left(l_{2}[x:=t]\right)\left(\lambda y^{l_{2}} \cdot p[x:=t]\right) \equiv$ $\equiv \mathbf{G}\left(l_{2}[x:=t]\right)\left(\lambda y^{l_{2}[x:=t]} \cdot p[x:=t]\right)$.

CASES $w \in \mathscr{H}_{G}, w \in \mathscr{G}_{G}$. Similarly.

Lemma 4.7. Let $c X_{1} \ldots X_{n}={ }_{\beta \eta} M$ for some constant $c$ and some $M \in \mathscr{O}_{G}$. Then $n \in\{1,2\}$ and $M \equiv c Y_{1} \ldots Y_{n}$ with $Y_{i}={ }_{\beta \eta} X_{i}$.

Proof. By Church-Rosser for $\beta \eta$-reduction.

In the definition of $\mathscr{G}_{G}$ one of the clauses is $A_{i} \mathscr{T}_{G}^{A_{i}}$ where one might expect the more general clause $l \mathscr{T}_{G}^{l}$. But $A_{i} \mathscr{T}_{G}^{A_{i}}$ is sufficient as follows from the next lemma.

LEMMA 4.8.

$$
l t^{l} \in \overline{\mathscr{O}_{G}} \text { for each } l \in \mathscr{L}_{G} .
$$

Proof. By induction on the structure of $l$. It is clear if $l=A_{1}, A_{2}$ or $P t^{A_{1}}$. So let $l=\mathbf{G} l_{1}\left(\lambda x^{l_{1}} \cdot l_{2}\right)$. Then $l t^{l}=\mathbf{G} l_{1}\left(\lambda x^{l_{1}} \cdot l_{2}\right) t^{l}=\Xi l_{1}\left(\mathbf{S}\left(\lambda x^{l_{1}} \cdot l_{2}\right) t^{l}\right)=\Xi l_{1}\left(\lambda x^{l_{1}} \cdot l_{2}\left(t^{l} x^{l_{1}}\right)\right)$. Now $t^{l} \in \mathscr{T}_{G}^{l}=\mathscr{T}_{G}^{G l_{1}\left(\lambda x^{l_{1}} . l_{2}\right)}$ and $x^{l_{1}} \in \mathscr{T}_{G}^{l_{1}}$, hence $t^{l} x^{l_{1}} \in \mathscr{T}_{G}^{l_{2}}$. So by the induction hypothesis one has $l_{2}\left(t^{l} x^{l_{1}}\right) \in \overline{\mathscr{O}_{G}}$. Hence $\Xi l_{1}\left(\lambda x^{l_{1}} \cdot l_{2}\left(t^{l} x^{l_{1}}\right)\right) \in \overline{\mathscr{O}_{G}}$.

LEMMA 4.9.

$$
l V \in \overline{\mathscr{O}_{G}} \Rightarrow V \in \overline{\mathscr{T}_{G}^{l}} \text {. }
$$

Proof. By induction on the structure of $l$. It is clear if $l=A_{1}, A_{2}$ or $P t^{A_{1}}$. So let $l=\mathbf{G} l_{1}\left(\lambda x^{l_{1}} \cdot l_{2}\right)$. Then $l V=\Xi l_{1}\left(\lambda x^{l_{1}} \cdot l_{2}\left(V x^{l_{1}}\right)\right)$. So $l_{2}\left(V x^{l_{1}}\right) \in \overline{\mathscr{O}_{G}}$ and hence by the induction hypothesis we have $V x^{l_{1}}=t^{l_{2}} \in \mathscr{T}_{G}^{l_{2}}$. So $V=\lambda x^{l_{1}} \cdot t^{l_{2}} \in \mathscr{T}_{G}^{l}$.

Now we will prove $\Gamma \vdash M, \Gamma \subseteq \overline{\mathscr{O}_{G}} \Rightarrow M \in \overline{\mathscr{O}_{G}}$. The proof is similar to the proof of Proposition B3.10, but without the induction loading. We used that induction loading to derive, in subcase $\Xi_{\mathrm{i}}(\mathrm{a})$, that if $\Gamma \vdash \Xi\left(\mathrm{K}_{p}\right)(\lambda x . O)$ then $x \notin \mathrm{FV}(O)$. But now we have $\Gamma \vdash \Xi(p)(\lambda x . O)$ where $x$ may occur free in $O$.

Proposition 4.10. $\quad \mathscr{O}_{G}$ is an invariant (modulo $\beta \eta$-equality) for derivations in $\mathscr{I} \mathbf{G}:$

$$
\Gamma \vdash M, \Gamma \subseteq \overline{\mathscr{O}_{G}} \Rightarrow M \in \overline{\mathscr{O}_{G}} .
$$

Proof. By induction on the derivation of $\Gamma \vdash M$. We only consider the three specific rules $\Xi_{\mathrm{e}}, \Xi_{\mathrm{i}}$ and $\mathrm{G}_{\mathrm{L}}$. (The two general rules are easy and $\mathrm{G}_{\mathrm{e}}, \mathrm{G}_{\mathrm{i}}$ are equivalent to $\Xi_{\mathrm{e}}, \Xi_{\mathrm{i}}$.)

CASE $\Xi_{\mathrm{e}} . \Gamma \vdash M$ is $\Gamma \vdash Y V$ as direct consequence of $\Gamma \vdash \Xi X Y, \Gamma \vdash X V$.

By the IH one has $\Xi X Y \in \overline{\mathscr{O}_{G}}, X V \in \overline{\mathscr{O}_{G}}$. From $\Xi X Y \in \overline{\mathscr{O}_{G}}$ it follows that $X=l, Y=$ $\lambda x^{l} . O$ where $O \in \mathscr{O}_{G}$. Now $X V=l V \in \overline{\mathscr{O}_{G}}$, hence $V=t^{l} \in \mathscr{T}_{G}^{l}$ by Lemma 4.9. So $M=\left(\lambda x^{l} . O\right) t^{l}=O\left[x^{l}:=t^{l}\right] \in \overline{\mathscr{O}_{G}}$ by Lemma 4.6. 
CASE $\Xi_{\mathrm{i}} . \quad \Gamma \vdash M$ is $\Gamma \vdash \Xi X Y$ as direct consequence of

$\Gamma \vdash \mathbf{L} X, \Gamma, X x \vdash Y x$ with $x \notin \mathrm{FV}(\Gamma, X, Y)$.

By the IH one has $\mathrm{L} X \in \overline{\mathscr{O}_{G}}$, hence $X=l \in \mathscr{L}_{G}$. Now $x$ is any variable, so we may assume that $x=x^{l} \in \mathscr{V}_{G}^{l}$. Then $l x \in \overline{\mathscr{O}_{G}}$ by Lemma 4.8 , hence by the IH we have $Y x \in \overline{\mathscr{O}_{G}}$. Let $Y x=O \in \overline{\mathscr{O}_{G}}$. Then $M=\Xi l\left(\lambda x^{l} . O\right) \in \overline{\mathscr{O}_{G}}$.

CASE $\mathrm{G}_{\mathrm{L}} . \quad \Gamma \vdash M$ is $\Gamma \vdash \mathbf{L}(\mathbf{G} X Y)$ as direct consequence of

$$
\Gamma \vdash \mathbf{L} X, \Gamma, X x \vdash \mathbf{L}(Y x) \text { with } x \notin \mathrm{FV}(\Gamma, X, Y) \text {. }
$$

By the IH one has $\mathrm{L} X \in \overline{\mathscr{O}_{G}}$, hence $X=l \in \mathscr{L}_{G}$. Again we may assume that $x=x^{l} \in \mathscr{Y}_{G}^{l}$. Then $l x \in \overline{\mathscr{O}_{G}}$, hence by the IH we have $\mathbf{L}(Y x) \in \overline{\mathscr{O}_{G}}$. So $Y x=l_{1} \in \mathscr{L}_{G}$. We get $\left.\mathbf{G} X Y=\mathbf{G} l\left(\lambda x^{l} . l_{1}\right)\right) \in \overline{\mathscr{L}_{G}}$. Hence $\mathbf{L}(\mathbf{G} X Y) \in \overline{\mathscr{G}_{G}} \subseteq \overline{\mathscr{O}_{G}}$.

The invariant $\mathscr{O}_{G}$ is more complicated than $\mathscr{O}$ and we cannot prove completeness directly using this invariant, as in in B3.11 for $\mathscr{I} \Xi$ relative to PRED. In fact we will use $\mathscr{O}_{G}$ in order to show in 4.11-4.20 that in each proof $\Gamma \vdash_{\mathscr{J} G} M$, where $\Gamma \subseteq \overline{\mathscr{O}}_{G}$, all cut formulas can be eliminated. Then using another invariant $\mathscr{O}_{1}$, similar to $\mathscr{O}$ we prove in Proposition 4.25 completeness of $\mathscr{I} \mathbf{G}$ for PRED in a way similar to the proof of completeness of $\mathscr{I} \Xi$ for PRED.

Cut elimination. First we define the $\Xi$-length $L_{\Xi}(O)$ for $O \in \overline{\mathscr{O}_{G}}$ (and $L_{\Xi}(l)$ for $l \in \mathscr{L}_{G}$ ) and state some lemma's, without the (trivial) proofs.

DEFINITION 4.11 ( $\Xi$-length $L_{\Xi}$ ).

$$
\begin{aligned}
& L_{\Xi}\left(\left(P t^{A_{1}}\right) t^{P t^{A_{1}}}\right)=L_{\Xi}(\mathbf{L} l)=L_{\Xi}\left(A_{i} t^{A_{i}}\right)=0, \\
& L_{\Xi}\left(\Xi l\left(\lambda x^{l} \cdot O\right)\right)=1+L_{\Xi}(l)+L_{\Xi}(O), \\
& L_{\Xi}\left(A_{i}\right)=L_{\Xi}\left(P t^{A_{1}}\right)=0, \quad L_{\Xi}\left(\mathbf{G} l_{1}\left(\lambda x^{l_{1}} . l_{2}\right)\right)=1+L_{\Xi}\left(l_{1}\right)+L_{\Xi}\left(l_{2}\right) .
\end{aligned}
$$

Lemma 4.12. (i) $X, Y \in \mathscr{L}_{G} \cup \mathscr{O}_{G}, X \rightarrow \beta \eta Y \Rightarrow L_{\Xi}(X)=L_{\Xi}(Y)$.

(ii) $X, Y \in \mathscr{L}_{G} \cup \mathscr{O}_{G}, X=_{\beta \eta} Y \Rightarrow L_{\Xi}(X)=L_{\Xi}(Y)$.

Definition 4.13. Let $M \in \overline{\mathscr{O}_{G}}, M=\beta \eta$. Then $L_{\Xi}(M)=L_{\Xi}(O)$.

LEMMA 4.14. $L_{\Xi}\left(l t^{l}\right)=L_{\Xi}(l)$.

Lemma 4.15. $X \in \mathscr{L}_{G} \cup \overline{\mathscr{O}_{G}}, t^{l} \in \mathscr{T}_{G}^{l} \Rightarrow L_{\Xi}\left(X\left[x^{l}:=t^{l}\right]\right)=L_{\Xi}(X)$.

LEMMA 4.16.

$$
\Xi X Y \in \overline{\mathscr{O}_{G}}, X V \in \overline{\mathscr{O}_{G}} \Rightarrow L_{\Xi}(X V)<L_{\Xi}(\Xi X Y) \& L_{\Xi}(Y V)<L_{\Xi}(\Xi X Y) .
$$

Proof. $\Xi X Y=\Xi l\left(\lambda x^{l} . O\right)$ and $V \in \overline{\mathscr{T}_{G}^{l}}$. Now use Lemma's 4.14 and 4.15.

Definition 4.17. A cut elimination is a proof reduction of the following form

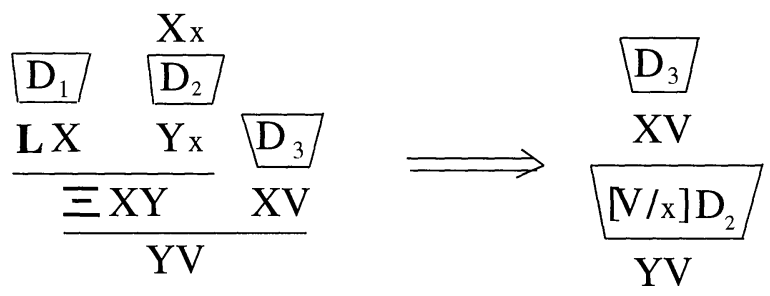


$\Xi X Y$ is called the cut formula.

LEMMA 4.18. In a proof of $\Gamma \vdash_{\mathscr{I}_{\mathrm{G}}} M\left(\right.$ or $\left.\Gamma \vdash_{\mathscr{G} \Xi} M\right)$, where $\Gamma \subseteq \overline{\mathscr{O}_{G}}$, all cut formulas can be eliminated by the above proof reduction.

Proof. By induction on the maximal $\Xi$-length of cut formulas in the proof of $\Gamma \vdash_{\mathscr{G}} M$. Consider a cut in the proof with a cut formula $\Xi X Y$ of maximal $\Xi-$ length. Eliminate this cut. Now new cut formulas may have been introduced at $X V$ and $Y V$, however by Lemma 4.16 these have shorter $\Xi$-length. In the same way all other cut formulas of maximal $\Xi$-length can be eliminated and so by the induction hypothesis all cut formulas can be eliminated.

REMARK 4.19. The above can easily be generalised to allow $\beta \eta$-equality steps (Eq-steps) as follows.

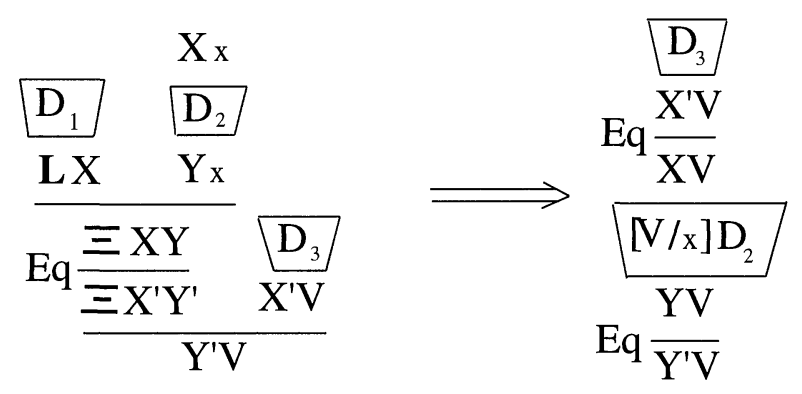

REMARK 4.20. Similarly we can define cut elimination for $\mathscr{I} \mathbf{P}$ as a proof reduction of the following form.
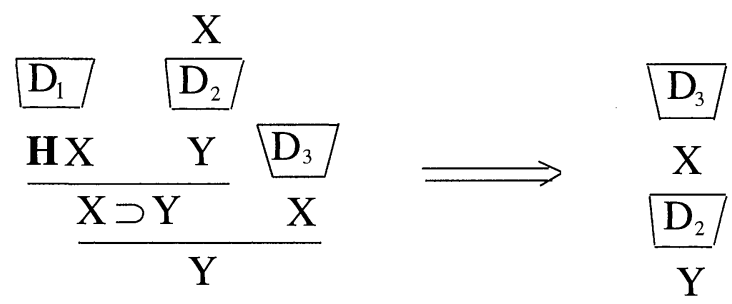

This is in fact a special case of Definition 4.17. (Substitute $\mathbf{K} X$ for $X$ and $\mathbf{K} Y$ for $Y$ in Definition 4.17.) Also in a proof of $\Gamma \vdash_{\mathscr{I P}} M$ where $\Gamma \subseteq \overline{\mathscr{O}_{G}}$ all cut formulas can be eliminated.

Now we define similar to the set $\mathscr{O}$ in B3.2 a set $\mathscr{O}_{1}$ such that $N \subseteq \overline{\mathscr{O}_{1}}$ for terms $N$ of interest occurring in a cut free proof of $\Gamma_{s}^{2,+},[\Delta]^{2}, \Gamma(\Delta, \varphi) \vdash_{\mathscr{I G}}[\varphi]^{2} M$.

DeFinition 4.21. Definition of $\mathscr{L}_{1}$.

$$
\mathscr{L}_{1} \equiv A_{1}\left|A_{2}\right| \mathscr{P}_{1}
$$


Definition of $\operatorname{sk}(l)$ and $\mathscr{V}_{1}^{l}$.

$$
\begin{aligned}
s k\left(A_{i}\right) & \equiv A_{i} \\
s k\left(P t^{A_{1}}\right) & \equiv P x^{A_{1}} \quad\left(\text { i.e., } s k\left(P t^{A_{1}}\right) \text { is independent of } t^{A_{1}}\right) \\
s k\left(\mathbf{G} l_{2}\left(\lambda x^{l_{2}} \cdot p_{1}\right)\right) & \equiv \mathbf{G}\left(s k\left(l_{2}\right)\right)\left(\lambda x^{l_{2}} \cdot s k\left(p_{1}\right)\right) . \\
\mathscr{V}_{1}^{l} & \equiv\left\{x_{1}^{l}, x_{2}^{l}, \ldots\right\}, x_{i}^{l}=x_{i}^{s k(l)}, x_{i}^{p_{1}} \neq x_{i}^{p_{2}} \text { if } \operatorname{sk}\left(p_{1}\right) \not \equiv \operatorname{sk}\left(p_{2}\right)
\end{aligned}
$$

Definition of $\mathscr{T}_{1}^{l}$.

$$
\begin{array}{rlrl}
\mathscr{T}_{1}^{l} & \equiv \mathscr{V}_{1}^{A_{1}}|a| f \mathscr{T}_{1}^{A_{1}} \mid g \mathscr{T}_{1}^{A_{1}} \mathscr{T}_{1}^{A_{2}} & & \text { if } l \equiv A_{1} ; \\
\mathscr{T}_{1}^{l} & \equiv \mathscr{V}_{1}^{A_{2}} & \text { if } l \equiv A_{2} ; \\
\mathscr{T}_{1}^{l} \equiv \mathscr{V}_{1}^{l} \mid \mathscr{T}_{1}^{\mathrm{G} l_{1}\left(\lambda x^{l_{1}} . l\right)} \mathscr{T}_{1}^{l_{1}} & \text { if } l \equiv P t^{A_{1}} \\
\mathscr{T}_{1}^{l} \equiv \mathscr{V}_{1}^{l}\left|\mathscr{T}_{1}^{\mathrm{G} l_{1}\left(\lambda x^{l_{1}} . l\right)} \mathscr{T}_{1}^{l_{1}}\right| \lambda x^{l_{2}} \mathscr{T}_{1}^{p_{1}} & & \text { if } \left.l \equiv \mathbf{G} l_{2}\left(\lambda x^{l_{2}} \cdot p_{1}\right)\right) .
\end{array}
$$

Definition of $\mathscr{P}_{1}, \mathscr{H}_{1}, \mathscr{G}_{1}$ and $\mathscr{O}_{1}$.

$$
\begin{aligned}
\mathscr{P}_{1} & \equiv P \mathscr{T}_{1}^{A_{1}} \mid \mathbf{G} l\left(\lambda x^{l} \cdot \mathscr{P}_{1}\right) ; \\
\mathscr{H}_{1} & \equiv\left(P t^{A_{1}}\right) t^{P^{A_{1}}} \mid \Xi l\left(\lambda x^{l} \cdot \mathscr{H}_{1}\right) ; \\
\mathscr{G}_{1} & \equiv \mathbf{L} \mathscr{L}_{1}\left|A_{i} \mathscr{T}_{1}^{A_{i}}\right| \Xi l\left(\lambda x^{l} \cdot \mathscr{G}_{1}\right) ; \\
\mathscr{O}_{1} & \equiv \mathscr{H}_{1} \mid \mathscr{G}_{1} .
\end{aligned}
$$

REMARKs 4.22. (i) Similar to Lemma $4.4(\mathrm{v})$ we now have $\mathscr{H}_{1}=\mathscr{P}_{1} \mathscr{T}_{1}^{\mathscr{P}_{1}}$.

(ii) If $l \equiv A_{i}$ in $\mathbf{G} l\left(\lambda x^{l} \cdot p_{1}\right)$ in the definition of $\mathscr{P}_{1}$, then $x^{l}$ may occur free in $p_{1}$; but if $l \equiv p$ then $x^{l} \notin \mathbf{F V}\left(p_{1}\right)$. So $\mathbf{G} l\left(\lambda x^{l} \cdot p_{1}\right)$ may be split into $\mathbf{G} A_{i}\left(\lambda x^{A_{i}} \cdot p_{1}\right)$ and $\mathbf{G} p\left(\mathbf{K} p_{1}\right)$ and similarly for $\Xi l\left(\lambda x^{l} . g\right)$ in the definition of $\mathscr{G}_{1}$.

(iii) Note that $\overline{\mathscr{O}_{1}} \subseteq \overline{\mathscr{O}_{G}}$, hence Lemma 4.18 remains valid if $\overline{\mathscr{O}_{G}}$ is replaced by $\overline{\mathscr{O}_{1}}$.

Lemma's 4.6-4.9 mutatis mutandis also hold for $\mathscr{O}_{1}$. Moreover lemma's corresponding to Lemma's B3.7-B3.9 hold. Replace $[-]^{1}$ by $[-]^{2}$ and in the lemma corresponding to B3.7 take $\mathscr{H}_{1}$ instead of $\mathscr{P}_{1}$.

Lemma 4.23. Let $p, p^{\prime} \in \mathscr{P}_{1}$. Then $p M=p^{\prime} M^{\prime} \Rightarrow p=p^{\prime}$.

Proof. Induction on $p$.

The following lemma corresponds to Proposition B3.10.

Lemma 4.24. Let $\Gamma \subseteq \overline{\mathscr{O}_{1}}$ and let $\Gamma \vdash_{\mathscr{I}_{\mathrm{G}}} N$ be a cut free proof. Then

(i) If the last applied rule in the proof is not the $\Xi_{\mathrm{i}}$-rule, nor the $\mathrm{G}_{\mathrm{L}}$-rule (modulo the $\beta \eta$-rule), then $N \in \overline{\mathscr{O}_{1}}$.

(ii) If $N=$ lt with $l \in \mathscr{L}_{1}, t \in \mathscr{T}_{G}^{l}$ then $N \in \overline{\mathscr{O}_{1}}$.

Proof. The proof of (i) and (ii) is by simultaneous induction on $\Gamma \vdash N$. The two general cases are easy, so we only consider Cases $\Xi_{\mathrm{i}}$, for (ii), and $\Xi_{\mathrm{e}}$. $Y x$.

CASE $\Xi_{\mathrm{i}} . \Gamma \vdash N$ is $\Gamma \vdash l t=\Xi X Y$ as direct consequence of $\Gamma \vdash \mathbf{L} X, \Gamma, X x \vdash$

Now $l t=\mathbf{G} l_{1}\left(\lambda x^{l_{1}} \cdot p\right) t=\Xi l_{1}\left(\lambda x^{l_{1}} \cdot p\left(t x^{l_{1}}\right)\right)=\Xi X Y$. The IH for $\Gamma, l_{1} x^{l_{1}} \vdash p\left(t x^{l_{1}}\right)$ yields $p\left(t x^{l_{1}}\right)=O \in \overline{\mathscr{O}_{1}}$. So $\Xi l_{1}\left(\lambda x^{l_{1}} \cdot p\left(t x^{l_{1}}\right)\right)=\Xi l_{1}\left(\lambda x^{l_{1}} \cdot O\right) \in \overline{\mathscr{O}_{1}}$. 
CASE $\Xi_{\mathrm{e}} . \Gamma \vdash N$ is $\Gamma \vdash Y V$ as direct consequence of $\Gamma \vdash \Xi X Y, \Gamma \vdash X V$.

By the induction hypothesis for $\Gamma \vdash \Xi X Y$ we have $\Xi X Y \in \overline{\mathscr{O}_{1}}$. So $\Xi X Y=\Xi l_{1}\left(\lambda x^{l_{1}} . O\right)$. The IH for $\Gamma \vdash l_{1} V$ yields $l_{1} V \in \overline{\mathscr{O}}_{1}$, hence $V \in \overline{\mathscr{T}_{1}^{l_{1}}}$. So $Y V=O\left[x^{l_{1}}:=V\right] \in \overline{\mathscr{O}_{1}} . \quad \dashv$

Proposition 4.25 (Completeness of $\mathscr{I} \mathbf{G}$ for PRED).

$$
\Gamma_{s}^{2,+},[\Delta]^{2}, \Gamma(\Delta, \varphi) \vdash_{\mathscr{G}}[\varphi]^{2} M \Rightarrow \Delta \vdash_{\operatorname{PRED}} \varphi .
$$

Proof. The proof resembles very much the proof of Proposition B3.11. Note that $\Gamma_{s}^{2,+} \subseteq \overline{\mathscr{G}}_{1}$ and $\Gamma(\Delta, \varphi) \subseteq \overline{\mathscr{G}}_{1}$. Hence it is sufficient to prove

$$
\Gamma_{g},[\Delta]^{2} \vdash_{\mathscr{I} G} N, \quad \Gamma_{g} \subseteq \overline{\mathscr{G}_{1}}, \quad N=[\varphi]^{2} M \Rightarrow \Delta \vdash_{\text {PRED }} \varphi .
$$

Write $\Gamma=\Gamma_{g},[\Delta]^{2}$. Then $\Gamma \subseteq \overline{\mathscr{O}}_{1}$. The proof of $(* *)$ is by induction on the derivation of $\Gamma \vdash_{\mathscr{F}_{\mathrm{G}}} N$. We may assume that the derivation is cut free because $\overline{\mathscr{O}_{1}} \subseteq \overline{\mathscr{O}_{G}}$.

CASE 1. $\Gamma \vdash N$ because $N \in \Gamma$.

$N=[\varphi]^{2} M$, so $N \notin \overline{\mathscr{G}_{1}}$, hence $N \in[\Delta]^{2}$. We get from Lemma 4.23 that $[\varphi]^{2}=[\psi]^{2}$ for some $\psi \in \Delta$. So $\varphi=\psi$ and hence $\varphi \in \Delta$. Therefore $\Delta \vdash_{\operatorname{PRED}} \varphi$.

CASE 2. $\Gamma \vdash N$ is a direct consequence of $\Gamma \vdash N^{\prime}$ and $N=N^{\prime}$.

Now $N^{\prime}=N=[\varphi]^{2} M$ and by the IH for $N^{\prime}$ one has $\Delta \vdash_{\text {PRED }} \varphi$.

CASE $\Xi_{\mathrm{e}} . \Gamma \vdash N$ is $\Gamma \vdash Y V$ as direct consequence of $\Gamma \vdash \Xi X Y, \Gamma \vdash X V$.

$\Gamma \vdash N$ is cut free, hence from Lemma 4.24 we get that $\Xi X Y \in \overline{\mathscr{O}_{1}}$. We distinguish 3 subcases.

SUBCASE $\Xi_{\mathrm{e}}(\mathrm{a}) . X=A_{i}, Y=\lambda x^{A_{i}} . h$. Now $h=p t^{p}=[\psi]^{2} t^{p}$ for some $p \in$ $\mathscr{P}_{1}, \psi \in \mathbb{F}_{\text {PRED }}$. We have $X V=A_{i} V \in \overline{\mathscr{O}_{1}}$ by Lemma 4.24. Therefore $V=t^{A_{i}}=$ $\left[t_{\mathbf{A}_{i}}\right]^{2}$. Now $N=[\varphi]^{2} M=Y V=\left(\lambda x^{A_{i}} \cdot[\psi]^{2} t^{p}\right)\left[t_{\mathbf{A}_{i}}\right]^{2}=[\psi]^{2}\left[x^{A_{i}}:=\left[t_{\mathbf{A}_{i}}\right]^{2}\right] t^{\prime}=$ $\left[\psi\left[x^{\mathbf{A}_{i}}:=t_{\mathbf{A}_{i}}\right]\right]^{2} t^{\prime}$ for some $t^{\prime}$. So $[\varphi]^{2} \equiv\left[\psi\left[x^{\mathbf{A}_{i}}:=t_{\mathbf{A}_{i}}\right]\right]^{2}$. Hence $\varphi \equiv \psi\left[x^{\mathbf{A}_{i}}:=\right.$ $t_{\mathbf{A}_{i}}$. $\Xi X Y=\Xi A_{i}\left(\lambda x^{A_{i}} \cdot p t^{p}\right)=\mathbf{G} A_{i}\left(\lambda x^{A_{i}} \cdot p\right) t^{\prime}$ (for some $\left.t^{\prime}\right)=\left[\forall x^{\mathbf{A}_{i}} \psi\right]^{2} t^{\prime}$. So we have by the IH $\Delta \vdash_{\text {PRED }} \forall x \mathbf{A}^{\mathbf{A}_{i}} \psi$. Hence $\Delta \vdash_{\text {PRED }} \psi\left[x^{\mathbf{A}_{i}}:=t_{\mathbf{A}_{i}}\right] \equiv \varphi$.

SuBCASE $\Xi_{\mathrm{e}}(\mathrm{b}) . X=p, Y=\lambda x^{p} \cdot h$. Now $Y=\lambda x^{p} \cdot p_{2} t^{p_{2}}$ where $x^{p} \notin \mathrm{FV}\left(p_{2}\right)$. Then $N=[\varphi]^{2} M=Y V=p_{2} t^{\prime}$ for some $t^{\prime}$. So $[\varphi]^{2}=p_{2}$. Let $p=\left[\varphi_{1}\right]^{2}$. Then $\Xi X Y=\mathbf{G} p\left(\mathbf{K} p_{2}\right) t^{\prime \prime}=\mathbf{F} p p_{2} t^{\prime \prime}=\left[\varphi_{1} \supset \varphi\right]^{2} t^{\prime \prime}$ for some $t^{\prime \prime}$. So we have by the IH

$$
\Delta \vdash_{\text {PRED }} \varphi_{1} \supset \varphi \text {. }
$$

Also $\Gamma \vdash X V=\left[\varphi_{1}\right]^{2} V$. So by the $\mathrm{IH}$ one has

$$
\Delta \vdash_{\mathrm{PRED}} \varphi_{1} \text {. }
$$

Therefore it follows by modus ponens that

$$
\Delta \vdash_{\text {PRED }} \varphi \text {. }
$$

SuBCASE $\Xi_{\mathrm{e}}(\mathrm{c}) . \Xi X Y=\Xi l\left(\lambda x^{l} . g.\right)$ This case is not applicable because $Y V \neq$ $[\varphi]^{2} M$.

CASE $\Xi_{\mathrm{i}} . \quad \Gamma \vdash N$ is $\Gamma \vdash \Xi X Y$ as direct consequence of

$$
\Gamma \vdash \mathbf{L} X, \Gamma, X x \vdash Y x \text { with } x \notin \mathrm{FV}(\Gamma, X, Y) \text {. }
$$


$\Xi X Y=[\varphi]^{2} M$. So $[\varphi]^{2}=\mathbf{G} l_{1}\left(\lambda x^{l_{1}} \cdot p_{2}\right)$ for some $l_{1} \in \mathscr{L}_{1}$ and $p_{2} \in \mathscr{P}_{1}$, and $\Xi X Y=\Xi l_{1}\left(\lambda x^{l_{1}} \cdot p_{2}\left(M x^{l_{1}}\right)\right)$. We consider 2 subcases.

SubCASE $\Xi_{\mathrm{i}}(\mathrm{a}) . X=A_{i}$. Let $p_{2}=[\psi]^{2}$. Then $\varphi=\forall x^{\mathbf{A}_{i}} \psi$. As $\Gamma, X x^{A_{i}} \vdash$ $p_{2}\left(M x^{A_{i}}\right)$ one has $\Delta \vdash_{\text {PRED }} \psi$ by the IH. Now $x^{A_{i}}$ does not occur in $\Gamma$, so

$$
\Delta \vdash_{\mathrm{PRED}} \forall x^{\mathbf{A}_{i}} \psi \text {. }
$$

SUBCASE $\Xi_{\mathrm{i}}(\mathrm{b}) . X=p_{1}$. Let $p_{1}=\left[\varphi_{1}\right]^{2}, p_{2}=\left[\varphi_{2}\right]^{2}$. Then $\varphi=\varphi_{1} \supset \varphi_{2}$. Now $\Gamma,\left[\varphi_{1}\right]^{2} x \vdash\left[\varphi_{2}\right]^{2}(M x)$. So one has by the IH $\Delta, \varphi_{1} \vdash$ PRED $\varphi_{2}$. Hence

$$
\Delta \vdash_{\mathrm{PRED}} \varphi_{1} \supset \varphi_{2} \text {. }
$$

CASE $\mathrm{G}_{\mathrm{L}} . \quad \Gamma \vdash N$ is $\Gamma \vdash \mathbf{L}(\mathbf{G} X Y Z)$ as direct consequence of

$$
\Gamma \vdash \mathbf{L} X, \Gamma, X x \vdash \mathbf{L}(Y x) \text { with } x \notin \mathrm{FV}(\Gamma, X, Y) \text {. }
$$

This case is not applicable because $\mathbf{L}(\mathbf{G} X Y Z) \neq[\varphi]^{2} M$.

§5. Completeness of $\mathscr{I} \mathbf{F}$ for PROP. We must show for $\varphi \in \mathbb{F}_{\mathrm{PROP}}, \Delta \subseteq$ $\mathbb{F}_{\text {PROP }}$ :

$$
\forall \text { closed } r \exists M \in \Lambda\left[[\Delta]_{r}^{2}, \Gamma_{r}^{2}(\Delta, \varphi) \vdash_{\mathscr{F}}[\varphi]_{r}^{2} M\right] \Rightarrow \Delta \vdash_{\mathrm{PROP}} \varphi .
$$

It is sufficient to show

$$
\exists M \in \Lambda\left[[\Delta]_{1}^{2}, \Gamma_{1}^{2}(\Delta, \varphi) \vdash_{\mathscr{F F}}[\varphi]_{1}^{2} M\right] \Rightarrow \Delta \vdash_{\mathrm{PROP}} \varphi .
$$

We omit I in the notation and we prove

$$
[\Delta]^{2}, \Gamma^{2}(\Delta, \varphi) \vdash_{\mathscr{F F}}[\varphi]^{2} M \Rightarrow \Delta \vdash_{\mathrm{PROP}} \varphi .
$$

The proof is much simpler than the proof of completeness of $\mathscr{I} \mathbf{G}$ for PRED, because there is no need for cut elimination. We define only one invariant $\mathscr{O}_{2}$ in 5.1, similar to $\mathscr{O}$ and $\mathscr{O}_{1}$ and using this invariant we prove the completeness in 5.2-5.5.

DefinITION 5.1 (Invariant for derivations in $\mathscr{I} \mathbf{F}$ ). $\mathscr{V}_{2}$ is a set of proposition variables. For every $p \in \mathscr{P}_{2}$ there is a different set $\mathscr{V}_{2}^{p}$ of variables.

Definition of $\mathscr{T}_{2}^{p}$.

$$
\begin{array}{ll}
\mathscr{T}_{2}^{p} \equiv \mathscr{V}_{2}^{p} \mid \mathscr{T}_{2}^{\mathbf{F} p_{1} p} \mathscr{T}_{2}^{p_{1}}, & \text { if } p \in \mathscr{V}_{2} ; \\
\mathscr{T}_{2}^{p} \equiv \mathscr{V}_{2}^{p}\left|\mathscr{T}_{2}^{\mathbf{F} p_{1} p} \mathscr{T}_{2}^{p_{1}}\right| \lambda x^{p_{2}} . \mathscr{T}_{2}^{p_{3}}, & \text { if } p \equiv \mathbf{F} p_{2} p_{3} .
\end{array}
$$

Definition of $\mathscr{P}_{2}, \mathscr{H}_{2}, \mathscr{G}_{2}$ and $\mathscr{O}_{2}$.

$$
\begin{aligned}
\mathscr{P}_{2} & \equiv \mathscr{V}_{2} \mid \mathbf{F} \mathscr{P}_{2} \mathscr{P}_{2} ; \\
\mathscr{H}_{2} & \equiv v t^{v}\left(v \in \mathscr{V}_{2}\right) \mid \Xi p_{1}\left(\lambda x^{p_{1}} \mathscr{H}_{2}\right) ; \\
\mathscr{G}_{2} & \equiv \mathbf{L} \mathscr{P}_{2} \mid \Xi \mathscr{P}_{2}\left(\mathbf{K} \mathscr{G}_{2}\right) ; \\
\mathscr{O}_{2} & \equiv \mathscr{H}_{2} \mid \mathscr{G}_{2} .
\end{aligned}
$$

REMARK 5.2. Similarly to Lemma $4.4(\mathrm{v})$ we have $\mathscr{H}_{2}=\mathscr{P}_{2} \mathscr{T}_{2}^{\mathscr{P}_{2}}$ modulo $\beta \eta$ equality. Note that in $\Xi p_{1}\left(\lambda x^{p_{1}} \cdot h\right)$ in the definition of $\mathscr{C}_{2}$ we have $h=p_{2} t^{p_{2}}$ where $x^{p_{1}} \notin \mathrm{FV}\left(p_{2}\right)$.

We have lemma's similar to Lemma's 4.6-4.9, with similar, but easier, proofs. $\left(\right.$ Now $\mathscr{L}_{2}=\mathscr{P}_{2}$.) 
Proposition 5.3. In $\mathscr{I} \mathbf{F}$ we have

$$
\Gamma \vdash M, \Gamma \subseteq \overline{\mathscr{O}_{2}} \Rightarrow M \in \overline{\mathscr{O}_{2}} .
$$

Proof. Similar to the proof of B3.10. Instead of Case $G_{L}$ we now have Case $F_{L}$.

LEMMA 5.4. (i) $\mathscr{G}_{2} \cap \mathscr{H}_{2}=\emptyset$;

(ii) $\overline{\mathscr{G}_{2}} \cap \overline{\mathscr{H}_{2}}=\emptyset$;

(iii) $[-]^{2}: \mathbb{F}_{\mathrm{PROP}} \rightarrow \mathscr{P}_{2}$ is a bijection.

(iv) Let $p, p^{\prime} \in \mathscr{P}_{2}$. Then $p M=p^{\prime} M^{\prime} \Rightarrow p=p^{\prime}$.

Proof. By easy inductions. For (ii) we use Church Rosser.

Proposition 5.5 (Completeness of $\mathscr{I F}$ for PROP).

$$
[\Delta]^{2}, \Gamma^{2}(\Delta, \varphi) \vdash_{\mathscr{I F}}[\varphi]^{2} M \Rightarrow \Delta \vdash_{\mathrm{PROP}} \varphi .
$$

Proof. Similar to the proof of Proposition 4.25 but easier. The subcases corresponding to subcases $\Xi_{\mathrm{e}}(\mathrm{a})$ and $\Xi_{\mathrm{i}}(\mathrm{a})$ do not occur.

$\S 6$. Completeness of $\mathscr{I} \mathbf{G}$ for PRED $\lambda \rightarrow$. In this section we will show completeness of $\mathscr{I} \mathbf{G}$ over predicate logic extended with higher type functions. In this logic we may quantify over all functions based on $\mathbf{A}_{1}$ and $\mathbf{A}_{2}$ and also over functions ranging over functions etc. and we can state e.g.,

$$
\forall f_{1} \in\left(\left(\mathbf{A}_{1} \rightarrow \mathbf{A}_{2}\right) \rightarrow \mathbf{A}_{1}\right) \forall f_{2} \in\left(\mathbf{A}_{1} \rightarrow \mathbf{A}_{2}\right)\left[\mathbf{P}\left(f_{1} f_{2}\right)\right] .
$$

DEFINITION 6.1. PRED $\lambda \rightarrow_{0}$, a preliminary version of Predicate Calculus with Higher Type Functions, is defined by

(i) Types of PRED $\lambda \rightarrow 0$

$$
\mathbb{L}_{\mathrm{PRED} \lambda \rightarrow_{0}}=\mathbf{A}_{1}\left|\mathbf{A}_{2}\right| \mathbb{L}_{\mathrm{PRED} \lambda \rightarrow 0} \rightarrow \mathbb{L}_{\mathrm{PRED} \lambda \rightarrow_{0}} .
$$

(ii) Variables of $\operatorname{PRED} \lambda \rightarrow_{0}$

$$
\mathbb{V}^{\mathbf{l}}=\left\{x_{1}^{\mathbf{l}}, x_{2}^{\mathbf{l}}, \ldots\right\} \quad \text { for } \mathrm{l} \in \mathbb{L}_{\operatorname{PRED} \lambda \rightarrow 0} .
$$

(iii) Terms of PRED $\lambda \rightarrow_{0}$

$$
\begin{aligned}
& \mathbb{T}^{\mathbf{l}}=\mathbf{a}\left|\mathbb{V}^{\mathbf{l}}\right| \mathbb{T}^{\mathbf{l}_{1} \rightarrow \mathbf{l}} \mathbb{T}^{\mathbf{l}_{1}}, \quad \text { if } \mathbf{l}=\mathbf{A}_{1} ;
\end{aligned}
$$

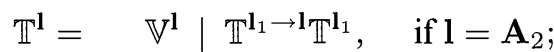

$$
\begin{aligned}
& \mathbb{T}^{\mathbf{l}}=\mathbf{f}\left|\mathbb{V}^{\mathbf{l}}\right| \mathbb{T}^{\mathbf{l}_{1} \rightarrow \mathbf{l}} \mathbb{T}^{\mathbf{l}_{1}}, \quad \text { if } \mathbf{l}=\mathbf{A}_{1} \rightarrow \mathbf{A}_{1} ; \\
& \mathbb{T}^{\mathbf{l}}=\mathbf{g}\left|\mathbb{V}^{\mathbf{l}}\right| \mathbb{T}^{\mathbf{l}_{1} \rightarrow \mathbf{l}} \mathbb{T}^{\mathbf{l}_{1}}, \quad \text { if } \mathbf{l}=\mathbf{A}_{1} \rightarrow\left(\mathbf{A}_{2} \rightarrow \mathbf{A}_{1}\right) ; \\
& \mathbb{T}^{\mathbf{l}}=\quad \mathbb{V}^{\mathbf{l}} \mid \mathbb{T}^{\mathbf{l}_{1} \rightarrow \mathbf{l}} \mathbb{T}^{\mathbf{l}_{1}}, \quad \text { if } \mathrm{l} \notin\left\{\mathbf{A}_{1}, \mathbf{A}_{2}, \mathbf{A}_{1} \rightarrow \mathbf{A}_{1}, \mathbf{A}_{1} \rightarrow\left(\mathbf{A}_{2} \rightarrow \mathbf{A}_{1}\right)\right\} .
\end{aligned}
$$

(iv) Well-formed formulas of $\operatorname{PRED} \lambda \rightarrow_{0}$

$$
\mathbb{F}_{\text {PRED } \lambda \rightarrow 0}=\mathbf{P} \mathbb{T}^{\mathbf{A}_{1}}\left|\mathbb{F}_{\text {PRED } \lambda \rightarrow 0} \supset \mathbb{F}_{\text {PRED } \lambda \rightarrow 0}\right|\left(\forall x^{\mathbf{l}}\right) \mathbb{F}_{\text {PRED } \lambda \rightarrow 0} .
$$

(v) Postulates of PRED $\lambda \rightarrow_{0}$ 


$$
\begin{aligned}
\varphi \in \Gamma & \Rightarrow \Gamma \vdash \varphi ; \\
\Gamma \vdash \varphi \supset \psi, \Gamma \vdash \varphi & \Rightarrow \Gamma \vdash \psi ; \\
\Gamma, \varphi \vdash \psi & \Rightarrow \Gamma \vdash \varphi \supset \psi ; \\
\Gamma \vdash \forall x^{1} \varphi, t \in \mathbb{T}^{1} & \Rightarrow \Gamma \vdash \varphi\left[x^{1}:=t\right] ; \\
\Gamma \vdash \varphi, x^{1} \notin \mathrm{FV}(\Gamma) & \Rightarrow \Gamma \vdash \forall x^{1} \varphi .
\end{aligned}
$$

DeFINITION 6.2. The mappings $[-]_{r}^{i}$ and $\Gamma$ in $\mathbf{B} 2.12$ from $\mathbb{F}_{\mathrm{PRED}}$ into $\Lambda^{s}(\Xi, \mathbf{L})$ and illative contexts respectively are extended to mappings from $\mathbb{F}_{\operatorname{PRED} \lambda \rightarrow 0}$ by altering some clauses and adding some clauses in the following way.

First we define $\left[\mathbf{A}_{j}\right]_{r}^{i}=A_{j}(j=1,2), \quad\left[\mathbf{l}_{1} \rightarrow \mathbf{l}_{2}\right]_{r}^{i}=\mathbf{F}\left[\mathbf{l}_{1}\right]_{r}^{i}\left[\mathbf{l}_{2}\right]_{r}^{i}$. We will denote $[\mathbf{l}]_{r}^{i}$ by $l$. Then we define

$$
\begin{aligned}
& {\left[x^{\mathbf{l}}\right]_{r}^{i}=x^{l}, \quad\left[\mathbf{t}_{1} \mathbf{t}_{2}\right]_{r}^{i}=\left[\mathbf{t}_{1}\right]_{r}^{i}\left[\mathbf{t}_{2}\right]_{r}^{i},} \\
& {\left[\forall x^{\mathbf{l}} \varphi\right]_{r}^{1}=\Xi l\left(\lambda x^{l} \cdot[\varphi]_{r}^{1}\right),\left[\forall x^{\mathbf{l}} \varphi\right]_{r}^{2}=\mathbf{G} l\left(\lambda x^{l} \cdot[\varphi]_{r}^{2}\right),} \\
& \Gamma\left(x^{\mathbf{l}}\right)=l x^{l}, \quad \Gamma\left(\forall x^{\mathbf{l}} \varphi\right)=\Gamma(\varphi)-\left\{l x^{l}\right\}, \quad \Gamma\left(\mathbf{t}_{1} \mathbf{t}_{2}\right)=\Gamma\left(\mathbf{t}_{1}\right) \cup \Gamma\left(\mathbf{t}_{2}\right), \\
& \Gamma_{r, s}^{1}=\left\langle\mathbf{L} A_{1}, \mathbf{L} A_{2}, \mathbf{F} A_{1} A_{1} f, \mathbf{F} A_{1}\left(\mathbf{F} A_{2} A_{1}\right) g, \mathbf{F} A_{1} \mathbf{H}(r \circ P), A_{1} a\right\rangle, \\
& \Gamma_{r, s}^{2}=\left\langle\mathbf{L} A_{1}, \mathbf{L} A_{2}, \mathbf{F} A_{1} A_{1} f, \mathbf{F} A_{1}\left(\mathbf{F} A_{2} A_{1}\right) g, \mathbf{F} A_{1} \mathbf{L}(r \circ P), A_{1} a\right\rangle, \\
& \Gamma_{r, s}^{i,+}=\Gamma_{r, s}^{i} \cup\left\{l x_{1}^{l} \mid l \neq A_{1}\right\}, \\
& {[-]^{i}, \Gamma_{s}^{i} \text { and } \Gamma_{s}^{i,+} \text { are obtained from }[-]_{r}^{i}, \Gamma_{r, s}^{i} \text { and } \Gamma_{r, s}^{i,+} \text { by everywhere omitting } r} \\
& \text { i.e., by replacing } r \circ P \text { by } P .
\end{aligned}
$$

Proposition 6.3. $\mathscr{I} \mathbf{G}$ is not complete for PRED $\lambda \rightarrow_{0}$.

Proof. Let $\varphi=\forall x_{1}^{\mathbf{A}_{1} \rightarrow \mathbf{A}_{1}} \forall x_{2}^{\mathbf{A}_{1}} P\left(x_{1} x_{2}\right) \supset \forall x_{2}^{\mathbf{A}_{1}} P x_{2}$. We will show

$$
\begin{gathered}
\forall \text { closed } r \exists M\left[\Gamma_{r, s}^{2,+}, \Gamma(\varphi) \vdash_{\mathscr{G}}[\varphi]_{r}^{2} M\right], \\
\nvdash_{\operatorname{PRED} \lambda \rightarrow 0} \varphi .
\end{gathered}
$$

Proof OF $(*)$. Let $X_{1}=\mathbf{G}\left(\mathbf{F} A_{1} A_{1}\right)\left(\lambda x_{1} \cdot \mathbf{G} A_{1}\left(\lambda x_{2} \cdot(r \circ P)\left(x_{1} x_{2}\right)\right)\right), Y_{1}=\mathbf{G} A_{1}\left(\lambda x_{2}\right.$. $\left.(r \circ P) x_{2}\right), M=\lambda x^{X_{1}} \cdot x^{X_{1}} I$ where $I=\lambda x^{A_{1}} \cdot x^{A_{1}}$. We will prove $(*)$ by showing $\forall$ closed $r\left[\Gamma_{r, s}^{2,+} \vdash_{\mathscr{I}_{\mathrm{G}}} \mathbf{F} X_{1} Y_{1} M\right]$.

Note that the rules $G_{e}$ and $G_{i}$ imply the rules $F_{e}$ and $F_{i}$. Let $r$ be a closed term in $\Lambda^{s}(\Xi, \mathbf{L})$. We have by rule $\mathrm{F}_{\mathrm{i}}$

$$
\mathbf{L} A_{1} \vdash \mathbf{F} A_{1} A_{1} I
$$

and from this we get by rule $\mathrm{G}_{\mathrm{e}}\left(\operatorname{taking} X=\mathbf{F} A_{1} A_{1}, Y=\lambda x_{1} . \mathbf{G} A_{1}\left(\lambda x_{2} \cdot(r \circ P)\left(x_{1} x_{2}\right)\right)\right.$, $\left.Z=x\left(=x^{X_{1}}\right), V=I\right)$

$$
\mathbf{L} A_{1}, X_{1} x \vdash Y_{1}(x I) .
$$

So we have

$$
\mathbf{L} A_{1}, X_{1} x \vdash Y_{1}(M x) .
$$

Moreover rule $F_{\mathrm{e}}$ yields

$$
\mathbf{L} A_{1}, \mathbf{F} A_{1} A_{1} x_{1}, A_{1} x_{2} \vdash A_{1}\left(x_{1} x_{2}\right) .
$$

Hence we get by applying rule $\mathrm{F}_{\mathrm{e}}$ once more

$$
\mathbf{L} A_{1}, \mathbf{F} A_{1} \mathbf{L}(r \circ P), \mathbf{F} A_{1} A_{1} x_{1}, A_{1} x_{2} \vdash \mathbf{L}\left((r \circ P)\left(x_{1} x_{2}\right)\right)
$$


and by rule $G_{L}$ twice

$$
\mathbf{L} A_{1}, \mathbf{F} A_{1} \mathbf{L}(r \circ P) \vdash \mathbf{L} X_{1} .
$$

From (1) and (2) we get by rule $\mathrm{F}_{\mathrm{i}}$

$$
\mathbf{L} A_{1}, \mathbf{F} A_{1} \mathbf{L}(r \circ P) \vdash \mathbf{F} X_{1} Y_{1} M .
$$

This proves $(*)$. But $\nvdash_{\mathrm{PRED} \lambda \rightarrow_{0}} \varphi$ because PRED $\lambda \rightarrow_{0}$ has no identity function in

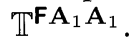

By similar examples one shows the need for other combinators or $\lambda$-terms. In fact all of these are needed in PRED $\lambda \rightarrow_{0}$ before completeness can be proved.

Definition 6.4 (Predicate Calculus with Higher Type Functions, PRED $\lambda \rightarrow$ ). Types and variables are as for PRED $\lambda \rightarrow_{0}$.

Terms of PRED $\lambda \rightarrow$

$$
\begin{aligned}
& \mathbb{T}^{\mathbf{l}}=\mathbf{a}\left|\mathbb{V}^{\mathbf{l}}\right| \mathbb{T}^{\mathbf{l}_{1} \rightarrow \mathbf{l}} \mathbb{T}^{\mathbf{l}_{1},}, \\
& \text { if } \mathbf{1}=\mathbf{A}_{1} \text {; } \\
& \mathbb{T}^{\mathrm{l}}=\mathbb{V}^{\mathrm{l}} \mid \mathbb{T}^{\mathrm{l}_{1} \rightarrow \mathrm{l}} \mathbb{T}^{\mathrm{l}_{1}}, \\
& \text { if } \mathbf{l}=\mathbf{A}_{2} \text {; } \\
& \mathbb{T}^{\mathbf{l}}=\mathbf{f}\left|\mathbb{V}^{\mathbf{l}}\right| \mathbb{T}^{\mathbf{l}_{1} \rightarrow \mathbf{l}} \mathbb{T}^{\mathbf{l}_{1}} \mid \lambda x^{\mathbf{A}_{1}} \cdot \mathbb{T}^{\mathbf{A}_{1}}, \\
& \text { if } \mathbf{l}=\mathbf{A}_{1} \rightarrow \mathbf{A}_{1} \text {; } \\
& \mathbb{T}^{\mathbf{l}}=\mathbf{g}\left|\mathbb{V}^{\mathbf{l}}\right| \mathbb{T}^{\mathbf{l}_{1} \rightarrow \mathbf{l}} \mathbb{T}^{\mathbf{l}_{1}} \mid \lambda x^{\mathbf{A}_{1}} \mathbb{T}^{\mathbf{A}_{2} \rightarrow \mathbf{A}_{1}}, \\
& \text { if } \mathbf{l}=\mathbf{A}_{1} \rightarrow\left(\mathbf{A}_{2} \rightarrow \mathbf{A}_{1}\right) \text {; } \\
& \mathbb{T}^{\mathbf{l}}=\mathbb{V}^{\mathbf{l}}\left|\mathbb{T}^{\mathbf{l}_{1} \rightarrow \mathbf{l}} \mathbb{T}^{\mathbf{l}_{1}}\right| \lambda x^{\mathbf{l}_{2}} \cdot \mathbb{T}^{\mathbf{l}_{3}}, \\
& \text { if } \mathbf{l}=\mathbf{l}_{2} \rightarrow \mathbf{l}_{3} \text {, } \\
& \mathbf{l} \notin\left\{\mathbf{A}_{1} \rightarrow \mathbf{A}_{1}, \mathbf{A}_{1} \rightarrow\left(\mathbf{A}_{2} \rightarrow \mathbf{A}_{1}\right)\right\} .
\end{aligned}
$$

The well-formed formulas of PRED $\lambda \rightarrow$ are defined as for PRED $\lambda \rightarrow_{0}$, but with the altered terms. The postulates of PRED $\lambda \rightarrow$ are as for PRED $\lambda \rightarrow_{0}$, plus

$$
\Gamma \vdash \varphi, \varphi=\beta \eta \psi \quad \Gamma \vdash \psi \text {. }
$$

REMARK 6.5. Ordinary predicate logic PRED can be expressed by the pure type system (PTS), see [1],

$$
\begin{array}{|ll}
\mathcal{S} & *^{p}, *^{s}, *^{f}, \square^{p}, \square^{s} \\
\mathscr{A} & *^{p}: \square^{p}, *^{s}: \square^{s} \\
\mathscr{R} & \left(*^{p}, *^{p}\right),\left(*^{s}, *^{p}\right),\left(*^{s}, \square^{p}\right) \\
& \left(*^{s}, *^{s}, *^{f}\right),\left(*^{s}, *^{f}, *^{f}\right)
\end{array}
$$

The point is that $*^{f}$ is a sort for function spaces like $\mathbf{A}_{1} \rightarrow \mathbf{A}_{2}$ or $\mathbf{A}_{1} \rightarrow\left(\mathbf{A}_{2} \rightarrow \mathbf{A}_{2}\right)$, with $\mathbf{A}_{1}, \mathbf{A}_{2} \in *^{s}$, but not for higher function spaces like $\left(\mathbf{A}_{1} \rightarrow \mathbf{A}_{2}\right) \rightarrow \mathbf{A}_{2}$. The system PRED $\lambda \rightarrow$ has higher function spaces and moreover it allows quantification over them. PRED $\lambda \rightarrow$ can be described more simply as the following PTS.

$$
\begin{array}{|c|}
\text { PRED } \lambda \rightarrow \\
\begin{array}{|ll}
\mathcal{S} & *^{p}, *^{s}, \square^{p}, \square^{s} \\
\mathscr{A} & *^{p}: \square^{p}, *^{s}: \square^{s} \\
\mathscr{R} & \left(*^{p}, *^{p}\right),\left(*^{s}, *^{p}\right),\left(*^{s}, \square^{p}\right),\left(*^{s}, *^{s}\right)
\end{array}
\end{array}
$$

Definition 6.6. We extend the mappings $[-]_{r}^{i}$ and $\Gamma$ of Definition 6.2 by

$$
\left[\lambda x^{\mathbf{l}_{1}} \cdot \mathbf{t}^{\mathbf{l}_{2}}\right]_{r}^{i}=\lambda x^{l_{1}} \cdot\left[\mathbf{t}^{\mathbf{l}_{2}}\right]_{r}^{i}, \quad \Gamma\left(\lambda x^{\mathbf{l}_{1}} \cdot \mathbf{t}^{\mathbf{l}_{2}}\right)=\Gamma\left(\mathbf{t}^{\mathbf{l}_{2}}\right)-\left\{l_{1} x^{l_{1}}\right\} .
$$


Proposition 6.7 (Soundness of the interpretations for PRED $\lambda \rightarrow$ ). Let $\Delta \cup\{\varphi\}$ $\subseteq \mathbb{F}_{\mathrm{PRED} \lambda \rightarrow}$, then the following hold for all closed $r$.

(i) $\Delta \vdash_{\mathrm{PRED} \lambda \rightarrow \varphi} \Rightarrow \Gamma_{r, s}^{1,+},[\Delta]_{r}^{1}, \Gamma(\Delta, \varphi) \vdash_{\mathscr{I} \Xi}[\varphi]_{r}^{1}$.

(ii) $\Delta \vdash_{\mathrm{PRED} \lambda \rightarrow \varphi} \Rightarrow \Gamma_{r, s}^{2,+},[\Delta]_{r}^{2}, \Gamma(\Delta, \varphi) \vdash_{\mathscr{I}_{\mathrm{G}}}[\varphi]_{r}^{2} M$ for some $M$.

PROOF. We shall not give the proof here. It is similar to the proof of Proposition B2.14 but longer because many more trivial cases have to be considered.

Now in 6.8-6.12 we shall prove completeness of $\mathscr{I} \mathbf{G}$ for PRED $\lambda \rightarrow$ under the indirect translation $[-]^{2}$. (Completeness of $\mathscr{I} \Xi$ for PRED $\lambda \rightarrow$ under the direct translation $[-]^{1}$ also holds, but we shall not give the proof here.)

First we define in Definition 6.8 an invariant $\mathscr{O}_{3}$, similar to $\mathscr{O}$ in B3.2 and $\mathscr{O}_{1}$ in Definition 4.21. We have $\mathscr{O}_{3} \subseteq \overline{\mathscr{O}_{G}}$, hence Lemma 4.18 yields that in a proof of $\Gamma \vdash_{\mathscr{I}_{\mathrm{G}}} M$ where $\Gamma \subseteq \overline{\mathscr{O}}_{3}$ all cut formulas can be eliminated. Then the proof of completeness of $\mathscr{I} \mathbf{G}$ for PRED $\lambda \rightarrow$ is similar to the proof of completeness of $\mathscr{I} \mathbf{G}$ for PRED in 4.22-4.25.

DeFINITION 6.8 (Invariant $\mathscr{O}_{3}$ for $\left.\mathscr{I} \mathbf{G}\right)$.

$$
\begin{aligned}
& \mathscr{L}_{3} \equiv A_{1}\left|A_{2}\right| \mathbf{F} \mathscr{L}_{3} \mathscr{L}_{3} \\
& \mathscr{Q}_{3} \equiv \mathscr{L}_{3} \mid \mathscr{P}_{3} \\
& \mathscr{V}_{3}^{l} \equiv\left\{x_{1}^{l}, x_{2}^{l}, \ldots\right\}, \quad x_{i}^{l_{1}} \neq x_{i}^{l_{2}} \text { if } l_{1} \neq l_{2} ; \\
& \mathscr{T}_{3}^{l} \equiv a\left|\mathscr{V}_{3}^{l}\right| \mathscr{T}_{3}^{\mathrm{F} l_{1} l} \mathscr{T}_{3}^{l_{1}}, \quad \text { if } l \equiv A_{1} ; \\
& \mathscr{T}_{3}^{l} \equiv \quad \mathscr{V}_{3}^{l} \mid \mathscr{T}_{3}^{\mathrm{Fl}_{1} l} \mathscr{T}_{3}^{l_{1}}, \quad \text { if } l \equiv A_{2} \text {; } \\
& \mathscr{T}_{3}^{l} \equiv f\left|\mathscr{V}_{3}^{l}\right| \mathscr{T}_{3}^{\mathrm{F} l_{1} l} \mathscr{T}_{3}^{l_{1}} \mid \lambda x^{A_{1}} \cdot \mathscr{T}_{3}^{A_{1}}, \quad \text { if } l \equiv \mathbf{F} A_{1} A_{1} ; \\
& \mathscr{T}_{3}^{l} \equiv g\left|\mathscr{V}_{3}^{l}\right| \mathscr{T}_{3}^{\mathrm{F} l_{1} l} \mathscr{T}_{3}^{l_{1}} \mid \lambda x^{A_{1}} \mathscr{T}_{3}^{\mathbf{F} A_{2} A_{1}} \text {, if } l \equiv \mathbf{F} A_{1}\left(\mathbf{F} A_{2} A_{1}\right) \text {; } \\
& \mathscr{T}_{3}^{l} \equiv \mathscr{V}_{3}^{l}\left|\mathscr{T}_{3}^{\mathrm{F} l_{1} l} \mathscr{T}_{3}^{l_{1}}\right| \lambda x^{l_{2}} \mathscr{T}_{3}^{l_{3}}, \quad \text { if } l \equiv \mathbf{F} l_{2} l_{3}, l \notin\left\{\mathbf{F} A_{1} A_{1}, \mathbf{F} A_{1}\left(\mathbf{F} A_{2} A_{1}\right)\right\} . \\
& \mathscr{P}_{3} \equiv P \mathscr{T}_{3}^{A_{1}} \mid \mathbf{G} q\left(\lambda x^{q} \cdot \mathscr{P}_{3}\right) \\
& \mathscr{H}_{3} \equiv\left(P t^{A_{1}}\right) t^{P t^{A_{1}}} \mid \Xi q\left(\lambda x^{q} \cdot \mathscr{H}_{3}\right) \text {; } \\
& \mathscr{G}_{3} \equiv \mathbf{L} \mathscr{Q}_{3}\left|A_{i} \mathscr{T}_{3}^{A_{i}}\right| \Xi q\left(\lambda x^{q} \cdot \mathscr{G}_{3}\right) ; \\
& \mathscr{O}_{3} \equiv \mathscr{H}_{3} \mid \mathscr{G}_{3} \\
& \mathscr{V}_{3}^{p} \equiv\left\{x_{1}^{p}, x_{2}^{p}, \ldots\right\}, x_{i}^{p}=x_{i}^{s k(p)}, x_{i}^{p_{1}} \neq x_{i}^{p_{2}} \text { if } s k\left(p_{1}\right) \not \equiv s k\left(p_{2}\right) ; \\
& s k(l) \equiv l \\
& \left.s k\left(P t^{A_{1}}\right) \equiv P x^{A_{1}} \quad \text { (i.e., } s k\left(P t^{A_{1}}\right) \text { is independent of } t^{A_{1}}\right) \text {; } \\
& s k\left(\mathbf{G} q\left(\lambda x^{q} \cdot p\right)\right) \equiv \mathbf{G}(s k(q))\left(\lambda x^{q} \cdot s k(p)\right) \\
& \mathscr{T}_{3}^{p} \equiv \mathscr{V}_{3}^{p} \mid \mathscr{T}_{3}^{\mathrm{G} q\left(\lambda x^{q} \cdot p\right)} \mathscr{T}_{3}^{q} \quad \text { if } p \equiv P t^{A_{1}} \\
& \left.\mathscr{T}_{3}^{p} \equiv \mathscr{V}_{3}^{p}\left|\mathscr{T}_{3}^{\mathbf{G} q\left(\lambda x^{q} \cdot p\right)} \mathscr{T}_{3}^{q}\right| \lambda x^{q_{1}} \cdot \mathscr{T}_{3}^{p_{1}} \text {, if } p \equiv \mathbf{G} q_{1}\left(\lambda x^{q_{1}} \cdot p_{1}\right)\right) \text {. }
\end{aligned}
$$

REMARKs 6.9. (i) Similar to Lemma $4.4(\mathrm{v})$ we now have $\mathscr{H}_{3}=\mathscr{P}_{3} \mathscr{T}_{3}^{\mathscr{P}_{3}}$ modulo $\beta \eta$-equality. 
(ii) If $q \equiv l$ in $\mathbf{G} q\left(\lambda x^{q} \cdot p_{1}\right)$ in the definition of $\mathscr{P}_{3}$, then $x^{q}$ may occur free in $p_{1}$; but if $q \equiv p$ then $x^{q} \notin \mathrm{FV}\left(p_{1}\right)$. So $\mathbf{G} q\left(\lambda x^{q} \cdot p_{1}\right)$ may be split into $\mathbf{G} l\left(\lambda x^{l} \cdot p_{1}\right)$ and $\mathbf{G} p\left(\mathbf{K} p_{1}\right)$ and similarly for $\Xi q\left(\lambda x^{q} \cdot g\right)$ in the definition of $\mathscr{G}_{3}$.

Lemma's 4.6-4.9 mutatis mutandis also hold for $\mathscr{O}_{3}$. (Replace $l$ by $q$.) Moreover lemma's corresponding to Lemma's B3.7-B3.9 hold. Replace $[-]^{1}$ by $[-]^{2}$ and in the lemma corresponding to B3.7 take $\mathscr{H}_{3}$ instead of $\mathscr{P}_{3}$.

Lemma 6.10. Let $p, p^{\prime} \in \mathscr{P}_{3}$. Then $p M=p^{\prime} M^{\prime} \Rightarrow p=p^{\prime}$.

ProOF. Induction on $p$.

Lemma 6.11. Let $\Gamma \subseteq \overline{\mathscr{O}_{3}}$ and let $\Gamma \vdash_{\mathscr{I}_{\mathrm{G}}} N$ be a cut free proof. Then

(i) If the last applied rule in the proof is not the $\Xi_{\mathrm{i}}$-rule, nor the $\mathrm{G}_{\mathrm{L}}$-rule (modulo the $\beta \eta$-rule), then $N \in \overline{\mathscr{O}_{3}}$.

(ii) If $N=$ q with $q \in \mathscr{Q}_{3}, t \in \mathscr{T}_{G}^{q}$ then $N \in \overline{\mathscr{O}_{3}}$.

Proof. Similar to the Proof of Lemma 4.24.

Proposition 6.12 (Completeness of $\mathscr{I} \mathbf{G}$ for PRED $\lambda \rightarrow$ relative to $[-]^{2}$ ).

$$
\Gamma_{s}^{2,+},[\Delta]^{2}, \Gamma(\Delta, \varphi) \vdash \mathscr{I G}[\varphi]^{2} M \Rightarrow \Delta \vdash_{\mathrm{PRED} \lambda \rightarrow} \varphi .
$$

Proof. We have $\mathscr{O}_{3} \subseteq \overline{\mathscr{O}}_{G}$, hence by Lemma 4.18 we may assume that the proof of $\Gamma_{s}^{2,+},[\Delta]^{2}, \Gamma(\Delta, \varphi) \vdash_{\mathscr{G}}[\varphi]^{2} M$ is cut free. Then the proof is similar to the proof of completeness of $\mathscr{I} \mathbf{G}$ for PRED in Proposition 4.25.

Remark 6.13 (Higher Order Predicate Calculus). Let us define PRED $\lambda \omega$ as PRED $\lambda \rightarrow$ but with

$$
\mathbb{L}_{\mathrm{PRED} \lambda \omega}=\mathbf{A}_{1}\left|\mathbf{A}_{2}\right| \mathbf{H} \mid \mathbb{L}_{\mathrm{PRED} \lambda \omega} \rightarrow \mathbb{L}_{\mathrm{PRED} \lambda \omega} .
$$

Thus PRED $\lambda \omega$ allows quantification over all functions, but also over all predicates and propositions. As a PTS the system PRED $\lambda \omega$ is $\operatorname{PRED} \lambda \rightarrow$ plus $\left(\square^{p}, *^{p}\right),\left(\square^{p}\right.$, $\left.\square^{p}\right)$. Given an extension of $\Gamma_{r, s}^{i}$ to include $\vdash \mathbf{L H}$, we would expect to prove the completeness of $\mathscr{I} \mathbf{G}$ over PRED $\lambda \omega$, but we have not yet succeeded. In particular we have not been able to prove cut elimination for $\mathbf{L H} \in \Gamma_{r, s}^{i}$, so the methods employed here are not all available.

§7. Conclusions and remarks. In [2] and in this paper we proved soundness and completeness for the direct and indirect interpretations of propositional and predicate calculus into illative systems:

(i) $\Delta \vdash_{\mathrm{PROP}} \varphi \Leftrightarrow \forall \operatorname{closed} r\left[\ldots 1,[\Delta]_{r}^{1} \vdash_{\mathscr{I P}_{\mathrm{P}}}[\varphi]_{r}^{1}\right]$;

(ii) $\Delta \vdash_{\mathrm{PRED}} \varphi \Leftrightarrow \forall$ closed $r\left[\cdots 1,[\Delta]_{r}^{1} \vdash \mathscr{I} \Xi[\varphi]_{r}^{1}\right]$;

(iii) $\Delta \vdash_{\mathrm{PROP}} \varphi \Leftrightarrow \forall$ closed $r \exists M\left[\cdots 2,[\Delta]_{r}^{2} \vdash \mathscr{I F}_{\mathrm{F}}[\varphi]_{r}^{2} M\right]$;

(iv) $\Delta \vdash_{\mathrm{PRED}} \varphi \Leftrightarrow \forall$ closed $r \exists M\left[\ldots 2,[\Delta]_{r}^{2} \vdash_{\mathscr{I}_{\mathrm{G}}}[\varphi]_{r}^{2} M\right]$;

where $\ldots i$ stands for $\Gamma_{r}^{i}(\Delta, \varphi)$ in the cases of PROP and for $\Gamma_{r, s}^{i,+}, \Gamma(\Delta, \varphi)$ in the cases of PRED.

REMARK 7.1. The proof of soundness $(\Rightarrow)$ was always simple. Completeness $(\Leftarrow)$ was proved each time by specialising to $r=\mathbf{I}$. This is a very natural choice, but one may wonder if the proofs can be given for other $r$, especially for $r=\mathrm{K}$. Inspecting the 4 proofs for $r=\mathbf{I}$ in this paper and in [2] one can verify that all these proofs need only minor changes to yield proofs for $r=\mathrm{K}$. 
Let us consider as an example the proof of completeness of $\mathscr{I} \mathbf{G}$ for PRED $(($ iv $) \Leftarrow)$ taking $r=\mathrm{K}$. Each $P t^{A_{1}}\left(t^{A_{1}} \in \mathscr{T}_{G}^{A_{1}}\right)$ in Section 4 must be replaced by $\mathrm{K}\left(P t^{A_{1}}\right)$. (And a resulting $\mathrm{K}\left(P t^{A_{1}}\right) M$ we rewrite $P t^{A_{1}}$ again.) Let us denote for the moment the resulting set $\mathscr{P}_{G}$ by $\mathscr{P}_{G K}$ and the set $\mathscr{P}_{G}$ of Section 4 for $r=1$ by $\mathscr{P}_{G \text { I. Then }}$. The

$$
\mathscr{P}_{G K}=\mathbf{K} \mathscr{P}_{G \mid} .
$$

In Definition 4.2 we define now

$$
\mathscr{T}_{G}^{l} \equiv \Lambda^{s}(\Xi, \mathbf{L}) \text { if } l \equiv \mathbf{K}\left(P t^{A_{1}}\right)
$$

As a result then $\mathscr{T}_{G}^{p}=\Lambda^{s}(\Xi, \mathbf{L})$ for each $p \in \mathscr{P}_{G K}$. These are nearly all changes that are needed to get a proof for $r=\mathbf{K}$ from the proof for $r=\mathbf{I}$ in Section 4 .

In another version of this paper, [3], we were not yet able to give completeness proofs of $\mathscr{I} \mathbf{G}$ for PRED and $\mathscr{I} \mathbf{F}$ for PROP $(($ iv $) \Leftarrow$ and $($ iii $) \Leftarrow)$ by specialising to $r=\mathbf{I}$. We there gave completeness proofs by taking $r=\mathrm{K}$, in a way different from the method sketched above. Let us consider now the proof of completeness of $\mathscr{I} \mathbf{G}$ for PRED in [3] taking $r=\mathbf{K}$. From

$$
[\varphi]_{\mathbf{K o l}}^{2}=\mathbf{K}[\varphi]_{1}^{1}
$$

in Lemma B2.15 we got

$$
[\varphi]_{\mathrm{K}}^{2} M=[\varphi]_{\mathrm{l}}^{1}
$$

Moreover in [3] we had a conservativity result

$$
\Gamma \vdash_{\mathscr{G}} M \Rightarrow \Gamma \vdash_{\mathscr{G} \Xi} M \text { for 'suitable' } \Gamma \text { and } M .
$$

Now the completeness proof in [3] was as follows

$$
\begin{array}{ll}
\Gamma_{\mathrm{K}, s}^{2,+},[\Delta]_{\mathrm{K}}^{2}, \Gamma(\Delta, \varphi) \vdash_{\mathscr{I}_{\mathrm{G}}}[\varphi]_{\mathrm{K}}^{2} M & \Rightarrow(\text { by } *) \\
\Gamma_{\mathbf{l}, s}^{1,+},[\Delta]_{\mathbf{l}}^{1}, \Gamma(\Delta, \varphi) \vdash_{\mathscr{G}}[\varphi]_{\mathbf{l}}^{1} \Rightarrow & (\text { by } * *) \\
\Gamma_{\mathbf{l}, s}^{1,+},[\Delta]_{\mathbf{l}}^{1}, \Gamma(\Delta, \varphi) \vdash_{\mathscr{I} \Xi}[\varphi]_{\mathbf{l}}^{1} \Rightarrow & (\text { by Proposition B3.14) } \\
\Delta \vdash_{\mathrm{PRED}} \varphi . &
\end{array}
$$

(To prove this conservativity result we had to show, in a way similar to the proof in the present paper, that the proof of $\Gamma \vdash_{\mathscr{G}} M$ could be made cut free.)

Also completeness of $\mathscr{I} \mathbf{F}$ relative to PROP for $[-]_{\mathbf{K}}^{2}$ followed from completeness of $\mathscr{I} \mathbf{P}$ relative to PROP for $[-]_{1}^{1}$ by a conservativity result for $\mathscr{I} \mathbf{F}$ relative to $\mathscr{I} \mathbf{P}$.

We also proved elsewhere completeness of $\mathscr{I} \mathbf{G}$ relative to PRED $\lambda \rightarrow$ for $[-]_{\mathrm{K}}^{2}$ Then completeness of $\mathscr{I} \Xi$ relative to PRED $\lambda \rightarrow$ for $[-]_{1}^{1}$ followed because system $\mathscr{I} \mathbf{G}$ is stronger then system $\mathscr{I} \Xi$. That last completeness is not proved in the present paper.

REMARK 7.2. We defined $\mathbf{P}, \mathbf{F}, \mathbf{G}$ in B2.3 in terms of the constant $\Xi$ and in B2.7 we obtained the following result.

The systems $\mathscr{I} \mathbf{P}, \mathscr{I} \Xi, \mathscr{I} \mathbf{F}$ and $\mathscr{I} \mathbf{G}$ are related as follows 


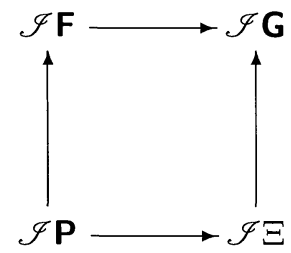

where $\longrightarrow$ denotes nondecreasing strength, i.e., $s_{1} \longrightarrow s_{2}$ means that for all $\Gamma, X$

$$
\Gamma \vdash_{s_{1}} X \Rightarrow \Gamma \vdash_{s_{2}} X \text {. }
$$

Moreover we had conservativity results like

$$
\Gamma \vdash_{\mathscr{F}_{\mathrm{G}}} M \Rightarrow \Gamma \vdash_{\mathscr{F} \Xi} M \text { for 'suitable' } \Gamma \text { and } M
$$

mentioned above. But in the proofs of soundness and completeness in the present paper we did not use these results. So one may wonder if soundness and completeness remain valid if we define $\mathbf{P}, \mathbf{F}$ and $\mathbf{G}$ to be constants. This is indeed the case (only the bases $\Gamma_{r, s}^{1}$ and $\Gamma_{r, s}^{2}$ must be rewritten). In the soundness proofs we did not use the fact that $\mathbf{P}, \mathbf{F}$ and $\mathbf{G}$ were defined in terms of $\Xi$. Also completeness remains valid because the systems with $\mathbf{P}, \mathbf{F}$ and $\mathbf{G}$ constant are less strong than the systems we considered. For example for system $\mathscr{I} \mathbf{F}$ then one does not have the relation $\mathbf{F}={ }_{\beta \eta} \lambda x y z . \Xi x(y \circ z)$.

REMARK 7.3. In B4.1 we stated that it is possible to work with variants of the systems $\mathscr{I} \mathbf{P}, \mathscr{I} \Xi, \mathscr{I} \mathbf{F}$ and $\mathscr{I} \mathbf{G}$ based on $\beta$-conversion only. This still holds. One may think that in the Proof of Lemma 4.9 (and the proof of Lemma 6.11) the $\eta$-axiom is used. This however is not the case, because by Definition 4.2 not only $f t^{A_{1}} \in \mathscr{T}_{G}^{A_{1}}$, but also $f \in \mathscr{T}_{G}^{\mathrm{F} A_{1} A_{1}}$ and similarly $g \in \mathscr{T}_{G}^{\mathrm{F} A_{1}\left(\mathbf{F} A_{2} A_{1}\right)}$.

\section{REFERENCES}

[1] H. Barendregt, Lambda calculi with types, Handbook of logic in computer science (S. Abramski, D. M. Gabbay, and T. S. E. Maibaum, editors), vol. II, Oxford University Press, 1992.

[2] H. Barendregt, M. Bunder, and W. DekKers, Systems of illative combinatory logic complete for first-order propositional and predicate calculus, this JOURNAL, vol. 58 (1993), pp. 769-788.

[3] W. DEKKERs, M. Bunder, and H. BARENDREGT, Completeness of two systems of illative combinatory logic for first-order propositional and predicate calculus, Archive for Mathematical Logic (1998), to appear.
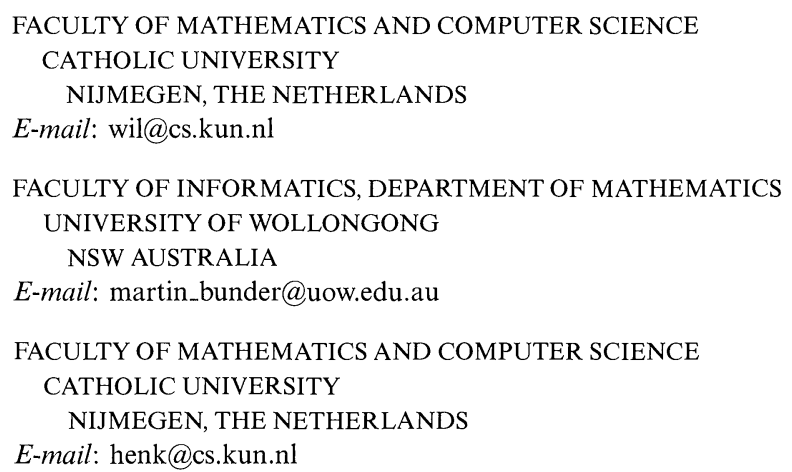